\title{
On Some Relations between Accretive, Positive, and Pseudocontractive Operators and Passivity Results in Hilbert Spaces and Nonlinear Dynamic Systems
}

\author{
M. De la Sen \\ Institute of Research and Development of Processes IIDP, Faculty of Science and Technology, University of the Basque Country, \\ P.O. Box 644 de Bilbao, Barrio Sarriena, Leioa, 48940 Bizkaia, Spain
}

Correspondence should be addressed to M. De la Sen; manuel.delasen@ehu.eus

Received 8 June 2017; Accepted 21 August 2017; Published 4 October 2017

Academic Editor: Seenith Sivasundaram

Copyright $\odot 2017$ M. De la Sen. This is an open access article distributed under the Creative Commons Attribution License, which permits unrestricted use, distribution, and reproduction in any medium, provided the original work is properly cited.

\begin{abstract}
This paper investigates some parallel relations between the operators $(I-G)$ and $G$ in Hilbert spaces in such a way that the pseudocontractivity, asymptotic pseudocontractivity, and asymptotic pseudocontractivity in the intermediate sense of one of them are equivalent to the accretivity, asymptotic accretivity, and asymptotic accretivity in the intermediate sense of the other operator. If the operators are self-adjoint then the obtained accretivity-type properties are also passivity-type properties. Such properties are very relevant in stability theory since they refer to global stability properties of passive feed-forward, in general, nonlinear, and time-varying controlled systems controlled via feedback by elements in a very general class of passive, in general, nonlinear, and time-varying controllers. These results allow the direct generalization of passivity results in controlled dynamic systems to wide classes of tandems of controlled systems and their controllers, described by G-operators, and their parallel interpretations as pseudocontractive properties of their counterpart $(I-G)$-operators. Some of the obtained results are also directly related to input-passivity, output-passivity, and hyperstability properties in controlled dynamic systems. Some illustrative examples are also given in the framework of dynamic systems described by extended square-integrable input and output signals.
\end{abstract}

\section{Introduction}

There is an important existing background literature available concerning passivity topics in dynamic systems. See, for instance, [1-6]. The passivity property in dynamic systems is closely related to that of positivity of the operator which describes the input-output behaviour of the system and it is a very general issue of global stability. In particular, the so-called Popov's hyperstability property of control systems has received a very important attention since it is basically related to the global closed-loop Lyapunov stability when (a) the feed-forward part of the control system (typically, the controlled system) is hyperstable and (b) the feedback part (typically, the controller) is any element belonging to a certain family of, in general, nonlinear and time-varying devices satisfying a hyperstability condition in terms of fulfilment of a Popov's type inequality $[7,8]$. Thus, the closedloop system is hyperstable if the controlled system and its controller are both hyperstable in the above senses. In the case when the controlled system is linear and time-invariant, its hyperstability property can be mathematically characterized by its transfer matrix being positive real which is closely related to the dissipativity and passivity (or positivity) of such a system and this translates in parallel in the feature that its associated input-output energy is nonnegative for all time irrespective of the controller under operation. It is wellknown that the asymptotic hyperstability formalism covers particular cases, the so-called Lure's and Popov's absolute stability problems. See, for instance, [1-15]. On the other hand, the so-called passivity property of dynamic systems can be described in the time-domain in terms of evolution of the so-called storage functions [6] and translates in the global Lypunov stability of all the feedback systems integrated by a feed-forward hyperstable controlled system and any controller belonging to the class of controllers satisfying a Popov's type inequality. Passivity of dynamic systems is also important since it relies on both conservative and dissipative systems. It has become apparent that such a property admits 
a precise characterization through constraints on the operators describing the feed-forward and feedback parts of the controlled dynamic system. On the other hand, there is also a rich literature on fixed point theory which is very related to convergence of sequences to fixed points and to convergence of trajectory solutions and sequences to equilibrium points, in general, when applied to dynamic systems. See, for instance, [16-22] and the abundant included background literature in those background references. In particular, the socalled pseudocontractions, asymptotic pseudocontractions, and asymptotic pseudocontractions in the intermediate sense in the framework of Hilbert spaces have also received an important attention along the last three decades. See [16-20] and references therein. On the other hand, some research on stability of topological stability of time-varying maps has been given in [23] while some results on stability of certain positive linear operators have been provided in [24]. Also, weaker-type contractive assumptions have been addressed in [25] in the context of metric and geodesic spaces and related "ad hoc" results have been obtained. See also $[26,27]$ and references therein concerning Ulam's type stability problems and stability conditions for switched dynamic systems.

By taking advantage of certain formally obtained relations of the pseudocontractive properties of an operator $(I-G)$ and the accretive properties of its counterpart operator $G$ in Hilbert spaces, the objective of this paper is to derive general conditions of the properties of accretivity, positivity, and passivity and their strict and asymptotic versions of an operator are asymptotically strictly pseudocontractive in the intermediate sense on a Hilbert space based on asymptotic pseudocontractive-type conditions on the operator $(I-G)$, the less restrictive asymptotic passivity conditions on $G$ being obtained if $(I-G)$ is asymptotically strictly, or strongly strictly pseudocontractive in the intermediate sense. The obtained results are applied for the Hilbert spaces of squareintegrable vector-valued functions so as to formulate general conditions on stability, hyperstability and passivity, and their asymptotic versions, of controlled dynamic systems formulated in the framework of such spaces. The passivity of the whole controlled conditions is decomposed on passivity-type conditions on both the controlled system and its controller. Note that the properties of passivity and hyperstability are very relevant properties in the field of dynamic systems since they are formulated jointly for classes of systems and controllers rather than for individual ones. Some illustrative examples are also given and discussed.

\section{Some Preliminaries}

Denote by $\mathbf{R}, \mathbf{R}_{+}$, and $\mathbf{R}_{0+}$ the sets of real, positive real, and nonnegative real numbers, respectively, and by $\mathbf{Z}, \mathbf{Z}_{+}$, and $\mathbf{Z}_{0+}$ the sets of real, positive real, and nonnegative integer numbers, respectively.

If the measurable function $f: \mathbf{i R}_{0+} \rightarrow \mathbf{C}^{n}$ then $f_{T}: \mathbf{R}_{+} \rightarrow$ C is the truncation of $f$ on $[0, T]$ defined by $f_{T}(\mathbf{i} t)=f(\mathbf{i} t)$ for $t \in[0, T]$ and $f_{T}(i t)=0$ for $t>T$ and any finite $T \in \mathbf{R}_{0+}$, where $\mathbf{i}=\sqrt{-1}$ is the imaginary complex unit.

$L_{p e}\left(\mathbf{i R}_{0+} ; \mathbf{C}^{n}\right)=\left\{f: \mathbf{i R}_{+} \rightarrow \mathbf{C}^{n}: f\right.$ is measurable and $\left.f_{T} \in L_{p} ; \forall T \in[0, \infty)\right\}$ is the $L_{p}\left(\mathbf{i R}_{0+} ; \mathbf{C}^{n}\right)$-space of the $p(\geq 1)$-integrable complex $n$-vector functions of imaginary complex argument. In the same way, we can define spaces of truncated functions $L_{p e}\left(\mathbf{R}_{0+} ; \mathbf{C}^{n}\right)$ and $L_{p e}^{n} \equiv L_{p e}\left(\mathbf{R}_{0+} ; \mathbf{R}^{n}\right)$ from $L_{p}\left(\mathbf{R}_{0+} ; \mathbf{C}^{n}\right)$ and $L_{p}^{n} \equiv L_{p}\left(\mathbf{R}_{0+} ; \mathbf{R}^{n}\right)$, respectively.

It is well known that $L_{p}\left(\mathbf{C} ; \mathbf{C}^{n}\right)$ endowed with an inner product $\langle\cdot, \cdot\rangle$ is a complete complex Hilbert space with norm $\|x\|=\left(\langle x, x\rangle^{p}\right)^{1 / p}$ (i.e., a Banach space with respect to the norm defined by such an inner product). We can extend from [1] the basic passivity concepts, of high relevance in stability, stabilization, and hyperstability problems of dynamic systems $[1-6,9]$, for real square-integrable operators to complex operators on $L_{2 e}\left(\mathbf{i R}_{0+} ; \mathbf{C}^{n}\right)$, leading to real nonnegative inner products, as follows in the subsequent definitions.

Definition 1. An operator $G: L_{2 e}\left(\mathbf{i R}_{0+} ; \mathbf{C}^{n}\right) \rightarrow L_{2 e}\left(\mathbf{i R}_{0+} ; \mathbf{C}^{n}\right)$ is said to be passive if

$$
\langle x, G x\rangle_{T} \geq 0, \quad \forall T \geq 0, \forall x \in L_{2 e},
$$

it is said to be strictly passive if there exists some real constant $\varepsilon>0$ such that

$$
\langle x, G x\rangle_{T} \geq \varepsilon\|x\|_{T 2}^{2}, \quad \forall T \geq 0, \forall x \in L_{2 e},
$$

and it is said to be strongly strictly passive if there exist some real constants $\varepsilon>0$ and $\delta>0$ such that

$$
\langle x, G x\rangle_{T} \geq \varepsilon\|x\|_{T 2}^{2}+\delta\|G x\|_{T 2}^{2}, \quad \forall T \geq 0, \forall x \in L_{2 e} .
$$

A strongly related concept to passivity is that of positivity. It is possible to extend the definition of positive operator [10] on Hilbert spaces to positive operators on the corresponding space of truncated functions.

Definition 2. An operator $G: L_{2 e}\left(\mathbf{i R}_{0+} ; \mathbf{C}^{n}\right) \rightarrow L_{2 e}\left(\mathbf{i R}_{0+} ; \mathbf{C}^{n}\right)$ is said to be positive if

$$
\langle G x, x\rangle_{T} \geq 0, \quad \forall T \geq 0, \forall x \in L_{2 e}
$$

and it is strictly positive if there exists some real constant $\varepsilon>0$ such that

$$
\langle G x, x\rangle_{T} \geq \varepsilon\|x\|_{T 2}^{2}, \quad \forall T \geq 0, \forall x \in L_{2 e} .
$$

It turns out that a strictly passive (resp., strictly positive) operator is also passive (resp., positive).

Proposition 3. Consider operators $G: L_{2 e}\left(\mathbf{i R}_{0+} ; \mathbf{C}^{n}\right) \rightarrow$ $L_{2 e}\left(\mathbf{i R}_{0+} ; \mathbf{C}^{n}\right)$ such that $\langle G x, x\rangle_{T}$ is real $\forall T \geq 0, \forall x \in L_{2 e}$. Then, the following properties hold.

(i) $G: L_{2 e}\left(\mathbf{i R}_{0+} ; \mathbf{C}^{n}\right) \rightarrow L_{2 e}\left(\mathbf{i R}_{0+} ; \mathbf{C}^{n}\right)$ is passive if and only if its adjoint operator $G^{*}: L_{2 e}\left(\mathbf{i R}_{0+} ; \mathbf{C}^{n}\right) \rightarrow L_{2 e}\left(\mathbf{i R}_{0+} ; \mathbf{C}^{n}\right)$ is positive and, in particular, $G: L_{2 e}\left(\mathbf{i R}_{0+} ; \mathbf{C}^{n}\right) \rightarrow L_{2 e}\left(\mathbf{i R}_{0+}\right.$; $\mathbf{C}^{n}$ ) is passive if and only if its transpose operator $G^{T}$ : $L_{2 e}\left(\mathbf{i R}_{0+} ; \mathbf{C}^{n}\right) \rightarrow L_{2 e}\left(\mathbf{i R}_{0+} ; \mathbf{C}^{n}\right)$ is positive.

(ii) If $G: L_{2 e}\left(\mathbf{i R}_{0+} ; \mathbf{C}^{n}\right) \rightarrow L_{2 e}\left(\mathbf{i R}_{0+} ; \mathbf{C}^{n}\right)$ is self-adjoint then it is positive if and only if it is passive (i.e., positivity and passivity are equivalent concepts for self-adjoint operators).

(iii) If $G: L_{2 e}\left(\mathbf{i R}_{0+} ; \mathbf{C}^{n}\right) \rightarrow L_{2 e}\left(\mathbf{i R}_{0+} ; \mathbf{C}^{n}\right)$ is positive then it is self-adjoint and passive. 
(iv) If $G: L_{2 e}\left(\mathbf{i R}_{0+} ; \mathbf{C}^{n}\right) \rightarrow L_{2 e}\left(\mathbf{i R}_{0+} ; \mathbf{C}^{n}\right)$ is symmetric then it is positive if and only if it is passive (i.e., positivity and passivity are equivalent concepts for real symmetric operators).

(v) $G^{*} G$ is positive and passive. If $G$ is normal then $G G^{*}$ is positive and passive for any $G: L_{2 e}\left(\mathbf{i R}_{0+} ; \mathbf{C}^{n}\right) \rightarrow$ $L_{2 e}\left(\mathbf{i R}_{0+} ; \mathbf{C}^{n}\right)$.

Proof. Property (i) is a direct consequence of Definitions 1 and 2. On the other hand, $G: L_{2 e}\left(\mathbf{i R}_{0+} ; \mathbf{C}^{n}\right) \rightarrow L_{2 e}\left(\mathbf{i R}_{0+} ; \mathbf{C}^{n}\right)$ is self-adjoint if and only if the inner product $\langle G x, x\rangle_{T}$ is real and $\langle G x, x\rangle_{T} \geq 0, \forall T \geq 0, \forall x \in L_{2 e}$. Then, the inner product $\langle G x, x\rangle_{T}$ is nonnegative real with $\langle G x, x\rangle_{T}=$ $\left\langle x, G^{*} x\right\rangle_{T}=\langle x, G x\rangle_{T} \geq 0, \forall T \geq 0, \forall x \in L_{2 e}$. So, $G$ : $L_{2 e}\left(\mathbf{i R}_{0+} ; \mathbf{C}^{n}\right) \rightarrow L_{2 e}\left(\mathbf{i R}_{0+} ; \mathbf{C}^{n}\right)$ is passive. From the same nonnegative real equalities, one concludes that if the operator is self-adjoint and passive then it is positive. Property (ii) is proved. Similarly, one concludes that if the operator is positive then it has to be self-adjoint and then passive, hence Property (iii). Property (iv) is a particular conclusion of the above ones for symmetric real operators. To prove Property (v), note that $\left\langle G^{*} G x, x\right\rangle_{T}=\langle G x, G x\rangle_{T}=\left\langle G x_{T}, G x_{T}\right\rangle=$ $\left\langle x, G^{*} G x\right\rangle=\|G x\|_{T 2}^{2} \geq 0 ; \forall x \in L_{2 e}\left(\mathbf{i R}_{0+} ; \mathbf{C}^{n}\right), \forall T \geq 0$, and $G^{*} G$ is positive and passive. If, in addition, $G$ is normal then $G G^{*}=G^{*} G$ so that $G G^{*}$ is positive and passive.

Proposition 4. Proposition 3 also holds "mutatis-mutandis" for Properties (i)-(v) if $G: L_{2 e}\left(\mathbf{i R}_{0+} ; \mathbf{C}^{n}\right) \rightarrow L_{2 e}\left(\mathbf{i R}_{0+} ; \mathbf{C}^{n}\right)$ is strictly passive/strictly positive.

Proposition 3 also holds "mutatis-mutandis" for Properties (i)-(v) if $G: L_{2 e}\left(\mathbf{i R}_{0+} ; \mathbf{C}^{n}\right) \rightarrow L_{2 e}\left(\mathbf{i R}_{0+} ; \mathbf{C}^{n}\right)$ is passive/positive and if it is strictly passive/strictly positive.

Roughly speaking, it is concluded from Propositions 3 and 4 that passivity (resp., strict passivity) and positivity (resp., strict positivity) are equivalent properties for complex self-adjoint and real symmetric operators. It is well-known that fixed point theory is a very useful tool to analyze stability and convergence problems in different applications, like, for instance, stability of continuous-time and discrete-time differential difference and hybrid equations, dynamic systems, and iterative computational processes. A main objective of this research is to discuss links between passivity properties versus pseudocontractive properties of operators in Hilbert spaces as well as generalize passivity bearing in mind the weaker pseudocontraction concept of that of pseudocontraction in the intermediate sense. See, for instance, [16-20]. Now, the passivity concepts for operators are related to those of pseudocontractions and pseudocontractions in the intermediate sense for alternative operators which are directly related to passive ones. The definition of accretive operators [16] can be applied to the space of truncated functions as follows.

Definition 5. Let $E$ be an arbitrary real Banach space endowed with a scalar product $\langle\cdot, \cdot\rangle$ from $E \times E$ to $\mathbf{R}$. An operator $U$ with domain $D(U)$ and range $R(U)$ in $E$ is called as follows:

(a) $K$-strictly accretive (or strictly accretive with constant $K)$ if, for each $x, y \in D(U)$, there is a $j(x-y) \in J(x-y)$, with $J$ being the normalized duality mapping, such that $\langle U x-$ $U y, j(x-y)\rangle \geq K\|x-y\|^{2}$ with $K>0$.
The operator $U$ is strictly accretive if some such a positive constant $K$ exists [16].

(b) Accretive if, for each $x, y \in D(U)$, there is $j(x-y) \in$ $J(x-y)$ such that, for each $x, y \in D(U),\langle U x-U y, j(x-y)\rangle \geq 0$ [16].

(c) $\left(\left\{K_{n}\right\}, K_{0}, K\right)$-asymptotically strictly accretive if there exist real constants $K_{0}>0$ and $K \geq 0$ and a real sequence $\left\{K_{n}\right\} \subset[0, \infty)$ satisfying $\liminf _{n \rightarrow \infty}\left(K_{n}\right) \geq K_{0}$ such that, for each $x, y \in D(U)$,

$$
\begin{aligned}
&\left\langle U^{n} y, U^{n} x, y-x\right\rangle \geq K_{n}\|x-y\| \\
&+K\left\|\left(I-U^{n}\right) y-\left(I-U^{n}\right) x\right\| ; \\
& \forall n \in \mathbf{Z}_{+} .
\end{aligned}
$$

The operator $U$ is asymptotically strictly accretive in the intermediate sense if some such a triple $\left(\left\{K_{n}\right\}, K_{0}, K\right)$ exists.

The operator $U$ is $\left(\left\{K_{n}\right\}, K_{0}, K\right)$-asymptotically strongly strictly accretive if it is $\left(\left\{K_{n}\right\}, K_{0}, K\right)$ asymptotically strictly accretive with $K>0$.

(d) $\left(\left\{K_{n}\right\}, K_{0}, K\right)$-asymptotically strictly accretive in the intermediate sense if there exist real constants $K_{0}>0$ and $K \geq 0$ and bounded real sequence $\left\{K_{n}\right\}$ satisfying $\liminf _{n \rightarrow \infty}\left(K_{n}\right) \geq K_{0}$ such that, for each $x, y \in D(U)$,

$$
\begin{gathered}
\liminf _{n \rightarrow \infty}\left(\left\langle U^{n} y-U^{n} x, y-x\right\rangle-K_{n}\|x-y\|\right. \\
\left.-K\left\|\left(I-U^{n}\right) y-\left(I-U^{n}\right) x\right\|\right) \geq 0 .
\end{gathered}
$$

The operator $U$ is asymptotically strictly accretive in the intermediate sense if some such a triple $\left(\left\{K_{n}\right\}, K_{0}, K\right)$ exists. The operator $U$ is $\left(\left\{K_{n}\right\}, K_{0}, K\right)$-asymptotically strongly strictly accretive in the intermediate sense if it is $\left(\left\{K_{n}\right\}, K_{0}, K\right)$ asymptotically strictly accretive in the intermediate sense with $K>0$.

We give now incremental-type concepts of incremental passivity and incremental positivity to be then related to the accretive property as follows. First, Definition 5 is extended to operators on $L_{2 e}\left(\mathbf{i R}_{0+} ; \mathbf{C}^{n}\right)$ as follows.

Definition 6. An operator $U: L_{2 e}\left(\mathbf{i R}_{0+} ; \mathbf{C}^{n}\right) \rightarrow L_{2 e}\left(\mathbf{i R}_{0+} ; \mathbf{C}^{n}\right)$ is as follows:

(a) Accretive if, provided that $\langle U x-U y, x-y\rangle_{T}$ is real for each $x, y \in D(U)$ and all $T \geq 0$, one has that, for each $x, y \in D(U)$ and somej $j(x-y) \in J(x-y)$, we have $\langle U x-$ $U y, j(x-y)\rangle_{T} \geq 0$.

(b) $U: L_{2 e}\left(\mathbf{i R}_{0+} ; \mathbf{C}^{n}\right) \rightarrow L_{2 e}\left(\mathbf{i R}_{0+} ; \mathbf{C}^{n}\right)$ is $K$-strictly accretive, if $\langle U x-U y, j(x-y)\rangle_{T} \geq K\|x-y\|_{T 2}^{2}$ for some positive real constant $K$ and $\forall T>0$.

(c) $U: L_{2 e}\left(\mathbf{i R}_{0+} ; \mathbf{C}^{n}\right) \rightarrow L_{2 e}\left(\mathbf{i R}_{0+} ; \mathbf{C}^{n}\right)$ is $\left(\left\{K_{n}\right\}, K_{0}, K\right)$ asymptotically strictly accretive if, for each $x, y \in D(U)$, there exist real constants $K_{0}>0$ and $K \geq 0$ and a real sequence $\left\{K_{n}\right\} \subset[0, \infty)$ satisfying $\liminf _{n \rightarrow \infty}\left(K_{n}\right) \geq K_{0}$ such that

$$
\begin{aligned}
& \left\langle U^{n} y, U^{n} x, y-x\right\rangle \\
& \geq K_{n}\|x-y\|_{T 2}^{2}+K\left\|\left(I-U^{n}\right) y-\left(I-U^{n}\right) x\right\|_{T 2}^{2} ; \\
& \forall n \in \mathbf{Z}_{+}, \forall T>0 .
\end{aligned}
$$


$U: L_{2 e}\left(\mathbf{i R}_{0+} ; \mathbf{C}^{n}\right) \rightarrow L_{2 e}\left(\mathbf{i R}_{0+} ; \mathbf{C}^{n}\right)$ is asymptotically strongly strictly accretive if it is asymptotically strictly accretive with $K>0$.

(d) $\left(\left\{K_{n}\right\}, K_{0}, K\right)$-asymptotically strongly strictly accretive in the intermediate sense if, for each $x, y \in D(U)$, there exist real constants $K_{0}>0$ and $K \geq 0$ and a bounded real sequence $\left\{K_{n}\right\}$ satisfying $\liminf _{n \rightarrow \infty}\left(K_{n}\right) \geq K_{0}$ such that

$$
\begin{aligned}
& \liminf _{n \rightarrow \infty}\left(\left\langle U^{n} y, U^{n} x, y-x\right\rangle-K_{n}\|x-y\|_{T 2}^{2}\right. \\
& \left.-K\left\|\left(I-U^{n}\right) x-\left(I-U^{n}\right) y\right\|_{T 2}^{2}\right) \geq 0, \quad \forall T>0 .
\end{aligned}
$$

$U: L_{2 e}\left(\mathbf{i} \mathbf{R}_{0+} ; \mathbf{C}^{n}\right) \rightarrow L_{2 e}\left(\mathbf{i} \mathbf{R}_{0+} ; \mathbf{C}^{n}\right)$ is asymptotically strongly strictly accretive in the intermediate sense if it is asymptotically strictly accretive in the intermediate sense with $K>0$.

The following definition is given extending the concepts of passivity to incremental passivity and to asymptotic incremental passivity in the intermediate sense.

Definition 7. An operator $G: L_{2 e}\left(\mathbf{i R}_{0+} ; \mathbf{C}^{n}\right) \rightarrow L_{2 e}\left(\mathbf{i R}_{0+} ; \mathbf{C}^{n}\right)$ is said to be incrementally passive if

$$
\langle x-y, G x-G y\rangle_{T} \geq 0, \quad \forall T \geq 0, \forall x, y \in L_{2 e},
$$

and it is incrementally strictly passive if there exists some real constant $\varepsilon>0$ such that

$$
\begin{aligned}
&\langle x-y, G x-G y\rangle_{T} \geq \varepsilon\|x-y\|_{T 2}^{2} \\
& \\
& \forall T \geq 0, \quad \forall x, y \in L_{2 e} .
\end{aligned}
$$

An operator $G: L_{2 e}\left(\mathbf{i R}_{0+} ; \mathbf{C}^{n}\right) \rightarrow L_{2 e}\left(\mathbf{i R}_{0+} ; \mathbf{C}^{n}\right)$ is said to be asymptotically incrementally strictly passive in the intermediate sense if there exists some real constant $\varepsilon>0$ such that

$$
\begin{aligned}
& \liminf _{n \rightarrow \infty}\left(\langle x-y, G x-G y\rangle_{T}-\varepsilon\|x-y\|_{T 2}^{2}\right. \geq 0, \\
& \forall T \geq 0, \forall x, y \in L_{2 e} .
\end{aligned}
$$

The counterpart definition to Definition 7 related to positivity follows.

Definition 8. An operator $G: L_{2 e}\left(\mathbf{i R}_{0+} ; \mathbf{C}^{n}\right) \rightarrow L_{2 e}\left(\mathbf{i R}_{0+} ; \mathbf{C}^{n}\right)$ is said to be incrementally positive if

$$
\langle G x-G y, x-y\rangle_{T} \geq 0, \quad \forall T \geq 0, \forall x, y \in L_{2 e},
$$

and it is incrementally strictly positive if there exists some real constant $\varepsilon>0$ such that

$$
\begin{aligned}
&\langle G x-G y, x-y\rangle_{T} \geq \varepsilon\|x-y\|_{T 2}^{2} \\
& \\
& \forall T \geq 0, \forall x, y \in L_{2 e} .
\end{aligned}
$$

An operator $G: L_{2 e}\left(\mathbf{i R}_{0+} ; \mathbf{C}^{n}\right) \rightarrow L_{2 e}\left(\mathbf{i R}_{0+} ; \mathbf{C}^{n}\right)$ is said to be incrementally strictly positive in the intermediate sense if there exists some real constant $\varepsilon>0$ such that

$$
\begin{aligned}
& \liminf _{n \rightarrow \infty}\left(\langle G x-G y, x-y\rangle_{T}-\varepsilon\|x-y\|_{T 2}^{2}\right) \geq 0, \\
& \forall T \geq 0, \forall x, y \in L_{2 e} .
\end{aligned}
$$

Remarks 9. It turns out that one has the following:

1. An incrementally strictly passive (resp., incrementally strictly positive) self-adjoint operator $G$ is also incrementally passive (resp., incrementally positive).

2. In case $G 0=0$, the accretive property (resp., the strict accretive property) is equivalent to incremental positivity (resp., strict positivity) and to the respective incremental passivity concepts for self-adjoint operators.

3. If $G 0=0$, incremental passivity and strict passivity are equivalent to passivity and strict passivity [1], respectively, and, furthermore, to positivity and strict positivity, respectively, if $G$ is self-adjoint.

4. If $G$ is self-adjoint with the same domain and codomain on a Hilbert space of finite dimension then the operator is Hermitian and in particular symmetric if the Hilbert space is real. In this case positivity, strict positivity, accretivity, and strict accretivity are equivalent to passivity, strict passivity, incremental passivity, and strict incremental passivity, respectively. If, in addition, $G 0=0$ then the respective incremental properties are equivalent to the standard ones.

Examples 10. Simple examples of some of the relevant previously introduced operators are now described.

1. The operator $T: X \rightarrow 2^{X}$ on a Banach space $X$ is strongly pseudocontractive if there exists $t>1$ such that, for all $x, y \in D(T)$ and $r>0$, the following inequality holds:

$$
\|(1+r)(x+y)-r t(T x-T y)\| \geq\|x-y\| .
$$

$T$ is pseudocontractive if the above inequality holds with $t=1$ [4].

2. The operator $T: X \rightarrow 2^{X}$ on a Banach space $X$ is strongly accretive if, for all $x, y \in D(T)$ and $r>0$, there exists $k>0$ such that the following inequality holds for some $k>0$ and all $x, y \in D(T)$ :

$$
\|(1-r k)(x-y)+r(T x-T y)\| \geq\|x-y\| .
$$

$T$ is accretive if the above inequality holds with $k=$ 0 , [4]. See $[4,28]$ and also [20] for the case of cyclic mappings.

3. A rational function $\widehat{h}(s)$ of the complex variable $s$ of real coefficients is positive real if (1) it is real for real $s$, (2) it has no poles in the open right half plane, (3) its poles $s=\mathbf{i} \omega$ at the imaginary axis, if any, are simple and their associate residues are simple, and (4) for all real $\omega$ such that $s=\mathbf{i} \omega$ is not a pole, $\operatorname{Re} \widehat{h}(\mathbf{i} \omega) \geq 0$. All these constraints together lead to $\operatorname{Re} \widehat{h}(s)>0$ for $\operatorname{Re} s \geq 0$.

4. Assume that such a positive real rational function $\widehat{h}(s)$ is a transfer function of a realizable linear timeinvariant system of one single input and one single output. That is, it has nonnegative relative degree 
(i.e., nonmore zeros than poles) so that it describes in Laplace transforms the input-output relation (i.e., the zero initial state response) of such a dynamic system. Then, the operator $\widehat{h}(s)$ is both passive and positive since it is self-adjoint by nature with $\widehat{h} 0=$ 0 and we can also say that the associated dynamic system is positive and passive. As a result, its inputoutput time integral is nonnegative for all time. A simple example is, for instance, $\hat{h}(s)=1 /(s+a)$ for $a \geq 0$ which is associated with the differential system $\dot{y}(t)=-a y(t)+u(t), y(0)=y_{0}$. If $a>0$ then the transfer function is strictly positive real (imaginary poles do not exist and the transfer function is stable satisfying also $\operatorname{Re} \widehat{h}(s)>0$ for $\operatorname{Re} s \geq 0$ and all $\omega \in(-\infty,+\infty))$, and the associated dynamic system is strictly passive. If the transfer function is modified to $\widehat{h}^{\prime}(s)=\widehat{h}(s)+d$ then $\operatorname{Re} \widehat{h}^{\prime}(s) \geq d$ for $\operatorname{Re} s \geq 0$ and all $\omega \in[-\infty,+\infty]$. The transfer function has a relative degree zero and it is said to be strongly strictly positive real (i.e., strictly positive real for any finite frequency and as frequency tends to \pm infinity) if $d>0$, with $d$ being a positive direct input-output interconnection gain in the dynamic system. Since the dynamic system is linear, the above properties imply also that it is incrementally passive and incrementally positive. See, for instance, $[3,4,7,29,30]$. The above examples are easily extendable to the discrete case, to the continuous-time and discrete-time multivariable cases (i.e., the cases when the output and/or the input can be vectors of dimensions greater than one), and also to dynamic systems of state dimensions being greater than one.

(a) It can be pointed out that the external positivity of a dynamic system in the sense that the solution trajectory solution (roughly speaking, the system output) is nonnegative for all time under arbitrary nonnegative initial conditions and nonnegative controls for all time is a different problem to the positivity and related passivity discussed here. Note that the positivity of the solution does not imply necessarily stability. Also, such an external positivity concept does not imply positivity for all time of the inputoutput energy for eventually negative controls. See, for instance, [31-33] and some references therein.

Some properties and relations for accretive operators on specific complex spaces are given and proved as follows.

Theorem 11. Assume that $U: L_{2 e}\left(\mathbf{i R}_{0+} ; \mathbf{C}^{n}\right) \rightarrow L_{2 e}\left(\mathbf{i R}_{0+} ; \mathbf{C}^{n}\right)$ is accretive; then the following properties hold for any $x, y \in$ $D(U)$ :

1. $\langle U x-U y, x-y\rangle_{T}+2\|U x-U y\|_{T 2} \geq 0, \forall T \geq 0$.

2. If, in addition, $U: L_{2 e}\left(\mathbf{i R}_{0+} ; \mathbf{C}^{n}\right) \rightarrow L_{2 e}\left(\mathbf{i R}_{0+} ; \mathbf{C}^{n}\right)$ is strictly accretive with constant $k$, odd superadditive, and bounded of norm $k_{U}<1 / 2$ then it is incrementally strictly positive with $k_{1}=k\left(1-2 k_{U}\right)$ and also incrementally strictly passive if the operator is self-adjoint.

3. Assume that $U 0=0$. If $U: L_{2 e}\left(\mathbf{i R}_{0+} ; \mathbf{C}^{n}\right) \rightarrow L_{2 e}\left(\mathbf{i R}_{0+}\right.$; $\mathrm{C}^{n}$ ) is accretive then $U$ is positive and, furthermore, passive if $U$ is self-adjoint. If $U: L_{2 e}\left(\mathbf{i R}_{0+} ; \mathbf{C}^{n}\right) \rightarrow$ $L_{2 e}\left(\mathbf{i R}_{0+} ; \mathbf{C}^{n}\right)$ is strictly accretive with constant $k$ and bounded of norm $k_{U}<1 / 2$ then it is strictly positive with $k_{1}=k\left(1-2 k_{U}\right)$ and, furthermore, it is strictly passive if the operator is self-adjoint.

\section{Properties (i)-(iii) hold "mutatis-mutandis" if $U$ :} $L_{2 e}\left(\mathbf{i R}_{0+} ; \mathbf{C}^{n}\right) \rightarrow L_{2 e}\left(\mathbf{i R}_{0+} ; \mathbf{C}^{n}\right)$.

Proof. Since $\|j(x-y)\|_{T}=\|(x-y)\|_{T}$ then by using Schwartz's inequality and the linearity properties of the Hilbert space,

$$
\begin{aligned}
\langle U x & -U y, x-y\rangle_{T}+2\|U x-U y\|_{T 2}\|x-y\|_{T 2} \\
\geq & \langle U x-U y, x-y\rangle_{T} \\
& +\|U x-U y\|_{T 2}\|j(x-y)+(x-y)\|_{T 2} \\
\geq & \langle U x-U y, x-y\rangle_{T} \\
& +\langle U x-U y, j(x-y)-(x-y)\rangle_{T} \\
= & \langle U x-U y, j(x-y)\rangle \geq k\|x-y\|_{T 2}^{2},
\end{aligned}
$$

and Property (i) follows by taking $k=0$ since $U: L_{2 e}\left(\mathbf{i R}_{0+}\right.$; $\left.\mathbf{C}^{n}\right) \rightarrow L_{2 e}\left(\mathbf{i} \mathbf{R}_{0+} ; \mathbf{C}^{n}\right)$ is strictly accretive. Also,

$$
\begin{aligned}
& \langle U x-U y, x-y\rangle_{T} \\
& \quad \geq\left(k\|x-y\|_{T 2}-2\|U x-U y\|_{T}\right)\|x-y\|_{T 2}
\end{aligned}
$$

If $U$ is strictly accretive, odd superadditive and bounded of norm $k_{U}<1 / 2$ then

$$
\begin{aligned}
& \langle U x-U y, x-y\rangle_{T}+2\|U(x-y)\|_{T}\|x-y\|_{T} \\
& \geq\langle U x-U y, x-y\rangle_{T} \\
& \quad+2\|U x+U(-y)\|_{T 2}\|x-y\|_{T 2} \geq k\|x-y\|_{T 2}^{2} .
\end{aligned}
$$

and $\langle U x-U y, x-y\rangle_{T} \geq k\left(1-2 k_{U}\right)\|x-y\|_{T 2}^{2}=k_{1} \| x-$ $y \|_{T 2}^{2}$ and Property (ii) is proved so that the identity mapping $j(x)=x$ fulfills the accretive property. Strict passivity/ incremental strict passivity for a self-adjoint operator follows from Proposition 4. The first part of Property (iii) follows from Property (i), and the second part follows from Property (ii) without requiring odd superadditivity and boundedness, if $U y=y=0$. Property (iv) is direct from Properties (i)-(iii) by changing the operator domain from $\mathbf{i} \mathbf{R}_{0+}$ to $\mathbf{R}_{0+}$. 
Definition 12. Let $H$ be a real Hilbert space $H$. Then, an operator $T$ from $D(T)$ (the Domain of $T$ ) to $R(T)$ (the Image of $T$ ) is as follows:

(a) $\left(K_{1}, K\right)$-pseudocontractive in the wide sense if there exist $K_{1}, K \in[0,1]$ such that

$$
\begin{aligned}
\|T x-T y\|^{2} \leq & K_{1}\|x-y\|^{2} \\
+K\|(I-T) x-(I-T) y\|^{2} ; & \\
& \forall x, y \in D(T) .
\end{aligned}
$$

The operator $T: D(T) \rightarrow R(T)$ is said to be pseudocontractive in the wide sense if such a pair $\left(K_{1}, K\right)$ exists.

The operator $T$ is nonexpansive if it is $(1,0)$ pseudocontractive in the wide sense.

(b) Pseudocontractive if

$$
\begin{array}{r}
\|T x-T y\|^{2} \leq\|x-y\|^{2}+\|(I-T) x-(I-T) y\|^{2} ; \\
\forall x, y \in D(T),
\end{array}
$$

and equivalently if

$$
\begin{aligned}
\langle T x-T y, x-y\rangle & \leq\|x-y\|^{2} \Longleftrightarrow \\
\langle(I-T) x-(I-T) y, x-y\rangle & \geq 0 ;
\end{aligned}
$$

$$
\forall x, y \in D(T)
$$

see $[16]$.

Note that if $T: D(T) \rightarrow R(T)$ is pseudocontractive in the wide sense it is pseudocontractive as well and a pseudocontraction in the wide sense with $K_{1}=K=1$ is equivalent to a pseudocontraction.

(c) K-strictly pseudocontractive if there exists a constant $K \in[0,1)$ such that

$$
\begin{array}{r}
\|T x-T y\|^{2} \leq\|x-y\|^{2}+K\|(I-T) x-(I-T) y\|^{2} ; \\
\forall x, y \in D(T) ;
\end{array}
$$

see [16].

The operator $T: D(T) \rightarrow R(T)$ is said to be strictly pseudocontractive if such a constant $K$ exists.

(d) $\left(\left\{K_{n}\right\}, K\right)$-asymptotically strictly pseudocontractive if there exists $K \in[0,1)$ and a sequence $\left\{K_{n}\right\} \subset[0, \infty)$ with $K_{n} \rightarrow 1$ as $n \rightarrow \infty$ such that

$$
\begin{aligned}
&\left\|T^{n} x-T^{n} y\right\|^{2} \leq K_{n}\|x-y\|^{2} \\
&+K\left\|\left(I-T^{n}\right) x-\left(I-T^{n}\right) y\right\|^{2} ; \\
& \quad \forall x, y \in D(T), \forall n(\geq 1) \in \mathbf{Z} .
\end{aligned}
$$

The operator $T: D(T) \rightarrow R(T)$ is said to be asymptotically strictly pseudocontractive if such a pair $\left(\left\{K_{n}\right\}, K\right)$ exists.

If $K=1$ then $T: D(T) \rightarrow R(T)$ is (nonstrictly) asymptotically pseudocontractive, and one has, equivalently (see [18]):

$$
\begin{aligned}
\left\langle T^{n} x-T^{n} y, x-y\right\rangle \leq & \frac{1+K_{n}}{2}\|x-y\|^{2} ; \\
& \forall x, y \in D(T), \forall n(\geq 1) \in \mathbf{Z} .
\end{aligned}
$$

(e) $\left(\left\{K_{n}\right\}, K_{0}, K\right)$-asymptotically strongly strictly pseudocontractive if there exist $K_{0}, K \in[0,1)$ and a sequence $\left\{K_{n}\right\} \subset$ $[0, \infty)$ with $K_{n} \rightarrow K_{0}$ as $n \rightarrow \infty$ such that

$$
\begin{aligned}
&\left\|T^{n} x-T^{n} y\right\|^{2} \leq K_{n}\|x-y\|^{2} \\
&+K\left\|\left(I-T^{n}\right) x-\left(I-T^{n}\right) y\right\|^{2} ; \\
& \forall x, y \in D(T), \forall n \in \mathbf{Z}_{+} .
\end{aligned}
$$

The operator $T: D(T) \rightarrow R(T)$ is said to be asymptotically strongly strictly pseudocontractive if such a triple $\left(\left\{K_{n}\right\}, K_{0}, K\right)$ exists. If $K=1$ then $T: D(T) \rightarrow R(T)$ is (nonstrictly) asymptotically pseudocontractive.

(f) Asymptotically nonexpansive if it is $\left(\left\{K_{n}\right\}, 0\right)$ asymptotically strictly pseudocontractive.

(g) $\left(\left\{K_{n}\right\}, K_{0}, K\right)$-asymptotically strongly strictly pseudocontractive in the intermediate sense if there exist $K_{0}, K \in$ $[0,1)$ and a real sequence $\left\{K_{n}\right\} \subset[0, \infty)$ satisfying $\left\{K_{n}\right\} \rightarrow K_{0}$ such that

$$
\begin{gathered}
\limsup _{n \rightarrow \infty} \sup _{x, y \in D(T)}\left(\left\|T^{n} x-T^{n} y\right\|^{2}-K_{n}\|x-y\|^{2}\right. \\
\left.-K\left\|\left(I-T^{n}\right) x-\left(I-T^{n}\right) y\right\|^{2}\right) \leq 0 .
\end{gathered}
$$

The operator $T: D(T) \rightarrow R(T)$ is said to be asymptotically strictly pseudocontractive in the intermediate sense if such a triple $\left(\left\{K_{n}\right\}, K_{0}, K\right)$ exists.

(h) $\left(\left\{K_{n}\right\}, K\right)$-asymptotically strictly pseudocontractive in the intermediate sense if there exists $K \in[0,1)$ and a real sequence $\left\{K_{n}\right\} \subset[0, \infty)$ satisfying $\left\{K_{n}\right\} \rightarrow 1$ such that

$$
\begin{gathered}
\limsup _{n \rightarrow \infty} \sup _{x, y \in D(T)}\left(\left\|T^{n} x-T^{n} y\right\|^{2}-K_{n}\|x-y\|^{2}\right. \\
\left.-K\left\|\left(I-T^{n}\right) x-\left(I-T^{n}\right) y\right\|^{2}\right) \leq 0 .
\end{gathered}
$$

The operator $T: D(T) \rightarrow R(T)$ is said to be asymptotically strictly pseudocontractive in the intermediate sense if such pair $\left(\left\{K_{n}\right\}, K\right)$ exists.

(i) $\left\{K_{n}\right\}$-asymptotically pseudocontractive in the intermediate sense if there exists a real sequence $\left\{K_{n}\right\} \subset[0, \infty)$ satisfying $\left\{K_{n}\right\} \rightarrow 1$ such that

$$
\begin{gathered}
\limsup _{n \rightarrow \infty} \sup _{x, y \in D(T)}\left(\left\|T^{n} x-T^{n} y\right\|^{2}-K_{n}\|x-y\|^{2}\right. \\
\left.-\left\|\left(I-T^{n}\right) x-\left(I-T^{n}\right) y\right\|^{2}\right) \leq 0 ;
\end{gathered}
$$

equivalently,

$$
\begin{aligned}
& \limsup _{n \rightarrow \infty} \sup _{x, y \in D(T)}\left(\left\langle T^{n} x-T^{n} y, x-y\right\rangle\right. \\
& \left.-\frac{1+K_{n}}{2}\|x-y\|^{2}\right) \leq 0 .
\end{aligned}
$$

The operator $T: D(T) \rightarrow R(T)$ is said to be asymptotically strictly pseudocontractive in the intermediate sense if such a sequence $\left\{K_{n}\right\}$ exists. 
(j) $\left(\left\{K_{n}\right\}, K_{0}\right)$-asymptotically strongly pseudocontractive in the intermediate sense if there exists $K_{0} \in[0,1)$ and a real sequence $\left\{K_{n}\right\} \subset[0, \infty)$ satisfying $\left\{K_{n}\right\} \rightarrow K_{0}$ such that

$$
\begin{gathered}
\limsup _{n \rightarrow \infty} \sup _{x, y \in D(T)}\left(\left\|T^{n} x-T^{n} y\right\|^{2}-K_{n}\|x-y\|^{2}\right. \\
\left.-\left\|\left(I-T^{n}\right) x-\left(I-T^{n}\right) y\right\|^{2}\right) \leq 0 .
\end{gathered}
$$

The operator $T: D(T) \rightarrow R(T)$ is said to be asymptotically strongly pseudocontractive in the intermediate sense if such a sequence $\left\{K_{n}\right\}$ exists.

The following result is obvious from Definition 12(g)-(i).

Proposition 13. If $T: D(T) \rightarrow R(T)$ is asymptotically strongly strictly pseudocontractive in the intermediate sense then it is asymptotically strictly pseudocontractive in the intermediate sense.

If $T: D(T) \rightarrow R(T)$ is asymptotically strictly pseudocontractive in the intermediate sense then it is asymptotically pseudocontractive in the intermediate sense.

If $T: D(T) \rightarrow R(T)$ is asymptotically strongly strictly pseudocontractive in the intermediate sense then it is asymptotically pseudocontractive in the intermediate sense.

If $T: D(T) \rightarrow R(T)$ is asymptotically strongly pseudocontractive in the intermediate sense then it is asymptotically pseudocontractive in the intermediate sense.

Theorem 14. Let $H$ be a real Hilbert space. Then, the following properties hold:

(i) Assume that $T: D(T) \rightarrow R(T)$ is $\left(K_{1}, K\right)$ pseudocontractive in the wide sense. Then $(I-T)$ is strongly accretive and it satisfies

$$
\begin{aligned}
& \langle(I-T) y-(I-T) x, y-x\rangle \\
& \quad \geq \frac{3-2 K-K_{1}}{3-K}\|x-y\|^{2} ; \quad \forall x, y \in D(T) .
\end{aligned}
$$

(ii) Assume that $T: D(T) \rightarrow R(T)$ is $K$-strictly pseudocontractive. Then $(I-T)$ is strongly accretive and it satisfies

$$
\begin{array}{r}
\langle(I-T) y-(I-T) x, y-x\rangle \geq \frac{1-K}{3-K}\|x-y\|^{2} ; \\
\forall x, y \in D(T) .
\end{array}
$$

(iii) Assume that $T: D(T) \rightarrow R(T)$ is pseudocontractive. Then, $(I-T)$ is accretive.

(iv) Assume that $T: D(T) \rightarrow R(T)$ is asymptotically strictly pseudocontractive. Then, $(I-T)$ is asymptotically strictly accretive.

(v) Assume that $T: D(T) \rightarrow R(T)$ is $\left(\left\{K_{n}\right\}, K_{0}, K\right)$ asymptotically strongly strictly pseudocontractive in the intermediate sense. Then, $(I-T)$ is asymptotically strongly strictly accretive in the intermediate sense satisfying for some $K_{0}$,
$K \in[0,1)$ and a convergent real sequence $\left\{K_{n}\right\} \subset[0, \infty)$ with $\left\{K_{n}\right\} \rightarrow K_{0}$ that

$$
\begin{aligned}
& \liminf _{n \rightarrow \infty}\left(\left\langle\left(I-T^{n}\right) y-\left(I-T^{n}\right) x, y-x\right\rangle\right. \\
& \left.-\frac{2-K-K_{0}}{3-K}\|x-y\|^{2}-\frac{1-K}{3-K}\left\|T^{n} y-T^{n} x\right\|^{2}\right) \\
& \geq 0 ; \quad \forall x, y \in D(T) .
\end{aligned}
$$

(vi) Assume that $T: D(T) \rightarrow R(T)$ is $\left(\left\{K_{n}\right\}, K\right)$-asymptotically strictly pseudocontractive in the intermediate sense. Then, $(I-T)$ is asymptotically strongly strictly accretive in the intermediate sense satisfying for some $K \in[0,1)$ and $a$ convergent real sequence $\left\{K_{n}\right\} \subset[0, \infty)$ with $\left\{K_{n}\right\} \rightarrow 1$ that

$$
\begin{array}{r}
\liminf _{n \rightarrow \infty}\left(\left\langle\left(I-T^{n}\right) y-\left(I-T^{n}\right) x, y-x\right\rangle\right. \\
\left.-\frac{1-K}{3-K}\|x-y\|^{2}-\frac{1-K}{3-K}\left\|T^{n} y-T^{n} x\right\|^{2}\right) \geq 0 ; \\
\forall x, y \in D(T) .
\end{array}
$$

(vii) Assume that $T: D(T) \rightarrow R(T)$ is $\left(\left\{K_{n}\right\}, K\right)$-asymptotically strongly pseudocontractive in the intermediate sense. Then, $(I-T)$ is asymptotically strictly accretive in the intermediate sense satisfying for some $K_{0} \in[0,1)$ and a convergent real sequence $\left\{K_{n}\right\} \subset[0, \infty)$ with $\left\{K_{n}\right\} \rightarrow K_{0}$ that

$$
\begin{aligned}
& \liminf _{n \rightarrow \infty}\left(\left\langle\left(I-T^{n}\right) y-\left(I-T^{n}\right) x, y-x\right\rangle\right. \\
& \left.-\frac{1-K_{0}}{2}\|x-y\|^{2}\right) \geq 0 ; \quad \forall x, y \in D(T) .
\end{aligned}
$$

Proof. Firstly, assume that, $\forall x, y \in D(T)$ is $\left(\left\{K_{n}\right\}, K\right)$-asymptotically strictly pseudocontractive, then one has for some sequence $\left\{\beta_{n}\right\} \subset \mathbf{R}_{+}$, with $\beta_{n}=\beta_{n}(x, y)$, that

$$
\begin{aligned}
\left\|T^{n} x-T^{n} y\right\|^{2}= & K_{n}\|x-y\|^{2} \\
& +K\left\|\left(I-T^{n}\right) x-\left(I-T^{n}\right) y\right\|^{2} \\
& -\beta_{n} ; \quad \forall n \in \mathbf{Z}_{+} \\
= & \|x-y\|^{2} \\
& +\left\|\left(I-T^{n}\right) x-\left(I-T^{n}\right) y\right\|^{2} \\
& -\alpha_{n}-\beta_{n} ; \quad \forall x, y \in D(T), \quad \forall n \in \mathbf{Z}_{+},
\end{aligned}
$$

where $\left\{\alpha_{n}\right\}$ is a real nonnegative sequence

$$
\begin{aligned}
\alpha_{n}= & \alpha_{n}(x, y) \\
= & \left(1-K_{n}\right)\|x-y\|^{2} \\
& +(1-K)\left\|\left(I-T^{n}\right) x-\left(I-T^{n}\right) y\right\|^{2} ; \\
& \forall n(\geq 1) \in \mathbf{Z}_{+} .
\end{aligned}
$$


Relation (38) is equivalent to

$$
\begin{aligned}
& \left\langle T^{n} y-T^{n} x, T^{n} y-T^{n} x\right\rangle \\
& =(x-y, x-y) \\
& \quad+\left\langle T^{n} x-T^{n} y-x+y, T^{n} x-T^{n} y-x+y\right\rangle \\
& \quad-\alpha_{n}-\beta_{n} \\
& =\langle y-x, y-x\rangle+\left\langle T^{n} x-T^{n} y, T^{n} x-T^{n} y\right\rangle \\
& \quad+\left\langle T^{n} x-T^{n} y, y-x\right\rangle+\left\langle y-x, T^{n} x-T^{n} y\right\rangle \\
& \quad+\langle y-x\rangle\langle y-x\rangle-\alpha_{n}-\beta_{n}=0 ; \\
& \quad \forall x, y \in D(T), \forall n \in \mathbf{Z}_{+},
\end{aligned}
$$

so that $2\left(\langle x-y, x-y\rangle-\left\langle T^{n} y-T^{n} x, y-x\right\rangle\right)-\alpha_{n}-\beta_{n}=0$ and then

$$
\begin{aligned}
& \left\langle T^{n} y-T^{n} x, y-x\right\rangle=\|x-y\|^{2}-\frac{\alpha_{n}+\beta_{n}}{2} \\
& \leq\|x-y\|^{2}-\frac{\alpha_{n}}{2}=\langle y-x, y-x\rangle-\frac{\alpha_{n}}{2} ; \\
& \forall x, y \in D(T), \forall n \in \mathbf{Z}_{+}, \\
& \text {or }\left\langle\left(I-T^{n}\right) y,\left(I-T^{n}\right) x, y-x\right\rangle \geq \frac{\alpha_{n}+\beta_{n}}{2} ; \\
& \forall x, y \in D(T), \forall n \in \mathbf{Z}_{+} .
\end{aligned}
$$

Then, one gets from (39)

$$
\begin{gathered}
\alpha_{n}=\left(1-K_{n}\right)\langle x-y, x-y\rangle+(1-K)\left\langle\left(I-T^{n}\right) x\right. \\
\left.-\left(I-T^{n}\right) y,\left(I-T^{n}\right) x-\left(I-T^{n}\right) y\right\rangle=\left(1-K_{n}\right) \\
\cdot\langle x-y, x-y\rangle+(1-K)\left\langle x-y+T^{n} y-T^{n} x, x\right. \\
\left.-y+T^{n} y-T^{n} x\right\rangle=\left(1-K_{n}\right)\langle y-x, y-x\rangle+(1 \\
-K)\langle y-x, y-x\rangle+(1-K)\left\langle x-y, T^{n} y-T^{n} x\right\rangle \\
+(1-K)\left\langle T^{n} y-T^{n} x, y-x\right\rangle+(1-K)\left\langle T^{n} y\right. \\
\left.-T^{n} x, T^{n} y-T^{n} x\right\rangle=\left(2-K-K_{n}\right)\langle y-x, y-x\rangle \\
-(1-K)\left\langle\left(I-T^{n}\right) y-\left(I-T^{n}\right) x, y-x\right\rangle+(1 \\
-K)\left\langle T^{n} y-T^{n} x, T^{n} y-T^{n} x\right\rangle ; \\
\forall x, y \in D(T), \forall n \in \mathbf{Z}_{+} .
\end{gathered}
$$

Now, one gets from (42) into (41) that

$$
\begin{aligned}
& \frac{3-K}{2}\left\langle\left(I-T^{n}\right) y-\left(I-T^{n}\right) x, y-x\right\rangle \\
& \geq \frac{2-K-K_{n}}{2}\langle x-y, x-y\rangle \\
& +\frac{1-K}{2}\left\langle T^{n} y-T^{n} x, T^{n} y-T^{n} x\right\rangle ; \\
& \forall x, y \in D(T), \forall n \in \mathbf{Z}_{+}
\end{aligned}
$$

so that

$$
\begin{aligned}
& \left\langle\left(I-T^{n}\right) y-\left(I-T^{n}\right) x, y-x\right\rangle \\
& \geq \frac{2-K-K_{n}}{3-K}\|x-y\|^{2}+\frac{1-K}{3-K}\left\|T^{n} y-T^{n} x\right\|^{2} ; \\
& \forall x, y \in D(T), \forall n \in \mathbf{Z}_{+} \text {, } \\
& \geq \frac{2-K-K_{n}}{3-K}\|x-y\|^{2} \\
& \forall x, y \in D(T), \forall n \in \mathbf{Z}_{+} .
\end{aligned}
$$

Property (i) follows from (44a) and (44b) with the replacement $T^{n} \rightarrow T$. Property (ii) follows from (44a) and (44b) with the replacement $T^{n} \rightarrow T$ in (44a) and (44b), the definition of strict pseudocontraction, and $K_{n}=1, \forall n(\geq) \in \mathbf{Z}_{+}$leading to $\left\langle\left(I-T^{n}\right) y,\left(I-T^{n}\right) x, y-x\right\rangle \geq(1-K) /(3-K)\|x-y\|^{2}$, $\forall x, y \in D(T)$, hence Property (ii). Properties (iii)-(iv) follow from Property (ii) with $K=1$ and $K \in[0,1)$. Property (v) follows with the replacement $\left\{T^{n}\right\} \rightarrow T$ in (44a) and (44b) and $\left\{K_{n}\right\} \rightarrow K_{0}$ with $K_{0}<1$. Property (vi) and Property (vii), respectively, follow with the replacement $\left\{T^{n}\right\} \rightarrow T$ in (44a) and (44b) and, respectively, $\left\{K_{n}\right\} \rightarrow 1$ and $K<1$, and $\left\{K_{n}\right\} \rightarrow K_{0}$ and $K=1$.

Recall that positivity is equivalent to passivity for selfadjoint operators and that accretivity can be interpreted as incremental positivity for inner products of pairs of elements in the operator domains and their respective images. The above result on pseudocontractions is now linked with some previous parallel positivity and passivity results from Proposition 3 and Theorems 11 and 14 on the extended space of truncated square-integrable vector functions.

Remark 15. Note from Theorems 14(v)-(vi) the important fact that if $T: D(T) \rightarrow R(T)$ is either $\left(\left\{K_{n}\right\}, K\right)$-asymptotically strictly pseudocontractive in the intermediate sense or $\left(\left\{K_{n}\right\}, K_{0}, K\right)$ asymptotically strongly strictly pseudocontractive in the intermediate sense then $(I-T)$ is in both cases asymptotically strongly strictly accretive in the intermediate sense.

The following two results follow from Theorem 14 .

Corollary 16. Let $H$ be a real Hilbert space. Then, the following properties hold:

(i) Assume that $T: D(T) \rightarrow R(T)$ is $\left(K_{1}, K\right)$-pseudocontractive and self-adjoint in the wide sense. Then, $(I-T)$ is $\bar{K}$-strongly accretive, $\bar{K}$-incrementally strictly passive, $\bar{K}$ incrementally strictly positive with $\bar{K}=\left(3-2 K-K_{1}\right) /(3-$ $K), \bar{K}$-strictly passive, and $\bar{K}$-strictly positive. If, in addition, $T 0=0$, then the incremental properties are equivalent to the nonincremental counterparts.

(ii) Assume that $T: D(T) \rightarrow R(T)$ is K-strictly pseudocontractive. Then $(I-T)$ is $\bar{K}^{\prime}$-strictly accretive with $\bar{K}^{\prime}=(1-$ $K) /(3-K)$. If, furthermore, $T: D(T) \rightarrow R(T)$ is self-adjoint then it is equivalently $\bar{K}^{\prime}$-incrementally strictly passive and $\bar{K}^{\prime}$ incrementally strictly positive. If, in addition, $T 0=0$, then 
the incremental properties are equivalent to the nonincremental counterparts.

(iii) Assume that $T: D(T) \rightarrow R(T)$ is $\left(\left\{K_{n}\right\}, K_{0}, K\right)$ asymptotically strongly strictly pseudocontractive in the intermediate sense and self-adjoint with $(I-T) \mathbf{0}=\mathbf{0}$ (equivalently with $T \mathbf{0}=\mathbf{0}$ if $(I-T)$ is linear $)$. Then, $(I-T)$ is $\left(\left\{\bar{K}_{n}\right\}, \bar{K}\right)$ asymptotically strictly passive (and, equivalently, asymptotically strictly positive) in the intermediate sense with $\bar{K}_{n}=\left(2-K-K_{n}\right) /(3-K), \forall n \in \mathbf{Z}_{+}$, such that $\bar{K}_{n} \rightarrow$ $\left(2-K-K_{0}\right) /(3-K)$ as $n \rightarrow \infty$ and $\bar{K}=(1-K) /(3-K)$ for some $K_{0}, K \in[0,1)$ and a convergent real sequence $\left\{K_{n}\right\} \subset[0, \infty)$ with $\left\{K_{n}\right\} \rightarrow K_{0}$.

The above result still holds with $K_{0}=1$ for some $K \in$ $[0,1)$, that is, $\left\{\bar{K}_{n}\right\} \rightarrow(1-K) /(3-K)$, if $T: D(T) \rightarrow$ $R(T)$ is $\left(\left\{K_{n}\right\}, K\right)$-asymptotically strictly pseudocontractive in the intermediate sense.

Proof. Properties (i) and (ii) follow from their counterparts of Theorem 14 and Property (iii) follows from Theorem 14(v) (see also Remarks 9).

Corollary 17. Let $H$ be a real Hilbert space. Then, the following properties hold:

(i) Assume that $T: D(T) \rightarrow R(T)$ is $\left(K_{1}, K\right)$-pseudocontractive in the wide sense. Then, the $\left(\left(3-2 K-K_{1}\right) /(3-K)\right)$ strongly accretive operator $(I-T)$ satisfies

$$
\begin{gathered}
\langle(I-T) T x-(I-T) x, T x-x\rangle \\
\geq \frac{3-2 K-K_{1}}{3-K}\|x-T x\|^{2} .
\end{gathered}
$$

(ii) Assume that $T: D(T) \rightarrow R(T)$ is K-strictly pseudocontractive. Then,

$$
\begin{array}{r}
\langle(I-T) T x-(I-T) x, T x-x\rangle \geq \frac{1-K}{3-K}\|x-T x\|^{2} \\
\forall x \in D(T) .
\end{array}
$$

(iii) Assume that $T: D(T) \rightarrow R(T)$ is $\left(\left\{K_{n}\right\}, K_{0}, K\right)$ asymptotically strongly strictly pseudocontractive in the intermediate sense for some $K, K_{0} \in[0,1)$ and $[0, \infty) \supset\left\{K_{n}\right\} \rightarrow$ $K_{0}$. Then,

$$
\begin{aligned}
\liminf _{n \rightarrow \infty}\left(\left\langle\left(I-T^{n}\right) T x-\left(I-T^{n}\right) x, T x-x\right\rangle\right. \\
-\frac{2-K-K_{n}}{3-K}\|x-T x\|^{2} \\
\left.\quad-\frac{1-K}{3-K}\left\|T^{n+1} x-T^{n} x\right\|^{2}\right) \geq 0 ; \quad \forall x \in D(T) .
\end{aligned}
$$

The result still holds with $K_{0}=1$ if $T: D(T) \rightarrow R(T)$ is $\left(\left\{K_{n}\right\}, K\right)$-asymptotically strictly pseudocontractive in the intermediate sense.

(iv) If $T: D(T) \rightarrow R(T)$ is linear then the commuting assumptions of Properties ( $i$ )-(iii) are removed.

(v) If $T: D(T) \rightarrow R(T)$ is linear and asymptotically strongly strictly pseudocontractive in the intermediate sense for some $K, K_{0} \in[0,1)$ and $[0, \infty) \supset\left\{K_{n}\right\} \rightarrow K_{0}$ and $T^{n}$ is pointwise convergent to $T_{0}: D(T) \rightarrow R(T)$ everywhere in its definition domain with $z=z(x)=\lim _{n \rightarrow \infty} T^{n} x=T_{0} x$ for any $x \in D(T)$ then

$$
\begin{aligned}
\liminf _{n \rightarrow \infty}\left(\left\langle T y-x+T_{0}(x-y), y-x\right\rangle\right. \\
\left.-\frac{2-K-K_{0}}{3-K}\|y-x\|^{2}-\frac{1-K}{3-K}\left\|T_{0}(y-x)\right\|^{2}\right) \\
\geq 0 ; \quad \forall x, y \in D(T) \\
\liminf _{n \rightarrow \infty}\left(\left\langle\left(\left(T^{2}-I\right)-T_{0}(T-I)\right) x,(T-I) x\right\rangle\right. \\
-\frac{2-K-K_{0}}{3-K}\|(T-I) x\|^{2} \\
\left.-\frac{1-K}{3-K}\left\|T_{0}(T-I) x\right\|^{2}\right) \geq 0 ; \quad \forall x \in D(T) .
\end{aligned}
$$

The result still holds with $K_{0}=1$ if $T: D(T) \rightarrow R(T)$ is $\left(\left\{K_{n}\right\}, K\right)$-asymptotically strictly pseudocontractive in the intermediate sense. Also, the result still holds with $K=1$ if $T: D(T) \rightarrow R(T)$ is asymptotically strongly pseudocontractive in the intermediate sense.

Proof. Properties (i) and (ii) follow from their counterparts of Theorem 14 while Property (iii) follows from Property (v) of Theorem 14, by fixing $y=T x$ for any given $x \in D(T)$. Property (iv) is direct if $T: D(T) \rightarrow R(T)$ is linear. Relation (45) of Property (v) follows from Properties (iii) and (iv), without equalizing $y$ to $T x$ for $x, y \in D(T), z(x)=$ $\lim _{n \rightarrow \infty} T^{n} x=\lim _{n \rightarrow \infty} T^{n+1} x=T_{0} x$ leading to

$$
\begin{aligned}
& \liminf _{n \rightarrow \infty}\left(\left\langle T y-x+T_{0} y-T_{0} x, y-x\right\rangle\right. \\
& \left.-\frac{2-K-K_{n}}{3-K}\|y-x\|^{2}-\frac{1-K}{3-K}\left\|T_{0} y-T_{0} x\right\|^{2}\right) \\
& \geq 0 ; \quad \forall x, y \in D(T),
\end{aligned}
$$

for any $x \in D(T)$ and the linearity of the limit operator $T_{0}$. Relation (46) follows from linearity and associated commuting property of $T_{0}$ and $T$ and (45) for $y=T x$ and $x \in$ $D(T)$.

Remark 18. Note that Theorem 14 and Corollaries 16 and 17 are directly applicable to operators defined on the extended real space $L_{2 e}\left(\mathbf{i R}_{0+} ; \mathbf{C}^{n}\right)$ of truncated functions on any real interval $[0, T]$ by defining on this space the scalar product $\langle\cdot, \cdot\rangle_{T}=\left\langle\cdot_{T},{ }_{T}\right\rangle$ for any real $T \in \mathbf{R}_{+}$. In particular, we have the subsequent result.

Corollary 19. Let $(I-G): L_{2 e}\left(\mathbf{i R}_{0+} ; \mathbf{C}^{n}\right) \rightarrow L_{2 e}\left(\mathbf{i R}_{0+} ; \mathbf{C}^{n}\right)$ be endowed with the scalar product on $L_{2 e}\left(\mathbf{i R}_{0+} ; \mathbf{C}^{n}\right)$ being everywhere real. Then, the following properties hold.

(i) Assume that $(I-G)$ is $\left(K_{1}, K\right)$-pseudocontractive on $L_{2 e}\left(\mathbf{i R}_{0+} ; \mathbf{C}^{n}\right)$ in the wide sense. Then $G$ is $\alpha$-strongly accretive with $\alpha=\left(3-2 K-K_{1}\right) /(3-K)$.

If, in addition, $G$ is odd superadditive and bounded of norm $k_{U}<1 / 2$ then it is strictly positive. 
If, furthermore, $G$ is self-adjoint then it is also incrementally strictly passive. If, in addition, $G 0=0$ then $G$ is also strictly positive and strictly passive.

(ii) Assume that $(I-G)$ is $K$-strictly pseudocontractive. Then $G$ is $\alpha$-strongly accretive with $\alpha=(1-K) /(3-K)$ and incrementally strictly positive.

If, in addition, $G$ is odd superadditive and bounded of norm $k_{U}<1 / 2$ then it is incrementally strictly positive.

If, furthermore, $G$ is self-adjoint then it is also incrementally strictly passive. If, in addition, $G 0=0$ then $G$ is also strictly positive and strictly passive.

(iii) Assume that $(I-G)$ is pseudocontractive. Then, $G$ is accretive and incrementally positive.

If, in addition, $G$ is self-adjoint then it is also incrementally passive. If, furthermore, $G 0=0$ then $G$ is also positive and passive.

(iv) Assume that $(I-G)$ is $\left(\left\{K_{n}\right\}, K\right)$-asymptotically strictly pseudocontractive. Then, $G$ is $\left(\left\{\bar{K}_{n}\right\}, \bar{K}_{1}\right)$-asymptotically strongly accretive with $[0, \infty) \ni \bar{K}_{n}=\left(2-K-K_{n}\right) /(3-K) \rightarrow$ $\bar{K}_{1}=(1-K) /(3-K)$ as $n \rightarrow \infty$, such that $[0, \infty) \supset\left\{K_{n}\right\} \rightarrow 1$ and $K \in[0,1)$, and incrementally asymptotically strictly positive. If, in addition, $G$ is self-adjoint then it is also incrementally asymptotically strictly passive. If, furthermore, $G 0=0$ then $G$ is also asymptotically strictly positive and asymptotically strictly passive.

(v) Assume that $(I-G)$ is $\left(\left\{K_{n}\right\}, K_{0}, K\right)$-asymptotically strongly strictly pseudocontractive in the intermediate sense. Then, $G$ is $\left(\left\{\bar{K}_{n}\right\}, \bar{K}_{0}, \bar{K}\right)$-asymptotically strongly accretive in the intermediate sense with $\bar{K}=(1-K) /(3-K)$ and $\left\{\bar{K}_{n}\right\} \rightarrow$ $\bar{K}_{0}=\left(2-K-K_{0}\right) /(3-K)$ such that $[0, \infty) \supset\left\{K_{n}\right\} \rightarrow$ $K_{0}(\in[0,1))$ and $K \in[0,1)$. If, in addition, $G$ is self-adjoint then it is also incrementally asymptotically strictly passive. If, furthermore, $G 0=0$ then $G$ is also asymptotically strictly positive and asymptotically strictly passive.

(vi) Assume that $(I-G)$ is $\left(\left\{K_{n}\right\}, K\right)$-asymptotically strictly pseudocontractive in the intermediate sense. Then, $G$ is $\left(\left\{\bar{K}_{n}\right\}, \bar{K}_{0}, \bar{K}\right)$-asymptotically strongly accretive in the intermediate sense with $\bar{K}=(1-K) /(3-K)$ and $\left\{\bar{K}_{n}\right\} \rightarrow \bar{K}_{0}=$ $(1-K) /(3-K)$ such that $[0, \infty) \supset\left\{K_{n}\right\} \rightarrow 1$ and $K \in[0,1)$. If, in addition, $G$ is self-adjoint then it is also incrementally asymptotically strictly passive.

Remark 20. It turns out that Corollary 19 is applicable to operators on $L_{2 e}\left(\mathbf{R}_{0+} ; \mathbf{R}^{p}\right)$ endowed with the same scalar product and of easy generalization to operators on $L_{p e}\left(\mathbf{R}_{0+} ; \mathbf{R}^{p}\right)$ for $p \in[1, \infty)$.

Proposition 21. Assume that $G: L_{2 e}\left(\mathbf{R}_{0+} ; \mathbf{R}^{p}\right) \rightarrow L_{2 e}\left(\mathbf{R}_{0+}\right.$; $\left.\mathbf{R}^{p}\right)$ is self-adjoint, one-to-one, of closed range, and strongly accretive. Then

1. $G: L_{2 e}\left(\mathbf{R}_{0+} ; \mathbf{R}^{p}\right) \rightarrow L_{2 e}\left(\mathbf{R}_{0+} ; \mathbf{R}^{p}\right)$ is asymptotically accretive, incrementally asymptotically positive, incrementally asymptotically passive, asymptotically positive, and asymptotically passive;

2. the operator $G^{n}$ on $L_{2 e}\left(\mathbf{R}_{0+} ; \mathbf{R}^{p}\right)$ is strictly accretive, incrementally strictly positive, incrementally strictly passive, asymptotically strictly positive, and strictly passive for any finite $n \in \mathbf{Z}_{+}$.

Proof. Note that if $G$ is an accretive operator on $L_{2 e}\left(\mathbf{R}_{+} ; \mathbf{R}^{p}\right)$ then $\langle G x-G y, x-y\rangle_{T} \geq 0$ for each $x, y \in D(G)$. Thus, $G^{n} x \in L_{2 e}\left(\mathbf{R}_{0+} ; \mathbf{R}^{p}\right)$ for any $G^{n} x \in L_{2 e}\left(\mathbf{R}_{0+} ; \mathbf{R}^{p}\right) ; \forall n \in \mathbf{Z}_{+}$, one has

$$
\begin{aligned}
\left\langle G^{2} x-G^{2} y, G x-G y\right\rangle_{T} \geq & \mu\left(G^{2}\right)\left|\langle G x-G y, x-y\rangle_{T}\right| \\
& =\mu\left(G^{2}\right)\langle G x-G y, x-y\rangle_{T} \\
& \geq \mu\left(G^{2}\right) \varepsilon_{g}\|x-y\|_{T 2}^{2} \\
& \vdots \\
\left\langle G^{n} x-G^{n} y, G^{n-1} x-G^{n-1} y\right\rangle_{T} & \geq \mu\left(G^{2^{n-1}}\right)\langle G x-G y, x-y\rangle_{T} \\
& \geq \mu\left(G^{2^{n-1}}\right) \varepsilon_{g}\|x-y\|_{T 2}^{2} \geq 0
\end{aligned}
$$

$\forall T>0$, with $\varepsilon_{g} \geq 0$ since $G$ is an accretive operator on $L_{2 e}\left(\mathbf{R}_{0+} ; \mathbf{R}^{p}\right)$, such that the real constant $\varepsilon_{g}>0$ if the operator $G$ is strongly accretive, where $\mu(G)>0$ is the minimum modulus of $G$ since it is one-to-one and of closed range, [10]. Now, (51) implies that $G: L_{2 e}\left(\mathbf{R}_{0+} ; \mathbf{R}^{p}\right) \rightarrow L_{2 e}\left(\mathbf{R}_{0+} ; \mathbf{R}^{p}\right)$ is asymptotically accretive, incrementally asymptotically positive, incrementally asymptotically passive (since the operator is self-adjoint), asymptotically positive, and asymptotically passive, since, in addition, the operator maps " 0 " into " 0 " and $\lim _{n \rightarrow \infty} \inf \mu\left(G^{n}\right) \geq 0$. On the other hand, for any finite $n \in \mathbf{Z}_{+}$, the composite operator $G^{n}$ on $L_{2 e}\left(\mathbf{R}_{0+} ; \mathbf{R}^{p}\right)$, resulting from composition of $G$ of $n$ times on itself, is strictly accretive, incrementally strictly positive, incrementally strictly passive, asymptotically strictly positive, and strictly passive, since, in addition, the operator maps " 0 " into " 0 " and $\mu\left(G^{n}\right)>0$ since $G^{n}$ on $L_{2 e}\left(\mathbf{R}_{0+} ; \mathbf{R}^{p}\right)$ is one-to-one and of closed range for any finite $n \in \mathbf{Z}_{+}$since $G$ is one-to-one and of closed range.

\section{Asymptotic Passivity in Dynamic Systems}

We first give some elementary concepts of usefulness to set the passivity framework. The notation for the spaces of real $n$-square-integrable and truncated $n$-square-integrable functions of nonnegative real domain is simplified due to subsequent extensive use as $L_{p}^{n} \equiv L_{p}\left(\mathbf{R}_{0+} ; \mathbf{R}^{n}\right)$ and $L_{p e}^{n}=$ $L_{p e}\left(\mathbf{R}_{0+} ; \mathbf{R}^{n}\right)$, respectively, for $p \in[1, \infty)$.

Consider a set $X$, a mapping $T: X \rightarrow X$, and a binary relation $R_{T} \subset X^{2}$ on $X$ defined by $T$ as $R_{T}=\{(x, T x): x \in$ $X$. A binary relation $R$ on $L_{p e}^{n}$ is said to be $L_{p}^{n}$-stable if $[x \in$ $\left.L_{p}^{n} \wedge(x, y) \in R\right] \Rightarrow y \in L_{p}^{n} . R$ is said to be $L_{p}$-stable with finite gain (wfg) if there exist finite nonnegative real constants $\gamma_{p}$ (gain) and $b_{p}$ (bias) such that

$$
\left[x \in L_{p}^{n} \wedge(x, y) \in R\right] \Longrightarrow\|y\|_{p} \leq \gamma_{p}\|x\|_{p}+b_{p}
$$

and $L_{p}^{n}$-stable with finite gain and zero bias $(w b)$ if $b_{p}=0$. Note that, if $R$ is $L_{p}^{n}$-stable wfg, then it is trivially $L_{p}^{n}$-stable wb since $y \in L_{p}^{n}$.

The following result generalizes a well-known passivity result of [11], also included in [1] in the context of a general 
framework setting on passivity, which is addressed based on some of the results given in the above section for asymptotic pseudocontractions in the intermediate sense. The result relies on the strict passivity of a tandem of dynamic systems consisting of a controlled system and its controller.

Theorem 22. Consider the real Hilbert space $L_{2}\left(\mathbf{R}_{0+} ; \mathbf{R}^{p}\right)$ endowed with the inner product $\langle\cdot, \cdot\rangle$. Assume that the selfadjoint operators $\left(I-G_{i}^{n}\right): L_{2 e}\left(\mathbf{R}_{0+} ; \mathbf{R}^{p}\right) \rightarrow L_{2 e}\left(\mathbf{R}_{0+} ; \mathbf{R}^{p}\right)$ for $i=1,2$ are invertible (i.e., one-to-one and of closed range) for $n \geq n_{0}$, some $n_{0} \in \mathbf{Z}_{+}$, and that $\left(I-G_{i}\right): L_{2 e}\left(\mathbf{R}_{0+} ; \mathbf{R}^{p}\right) \rightarrow$ $L_{2 e}\left(\mathbf{R}_{0+} ; \mathbf{R}^{p}\right)$ for $i=1,2$ satisfies $\left(I-G_{i}^{n}\right) 0=0$ for $n \geq n_{0}$ (if both operators are linear, it suffices that $\left.G_{i}^{n} 0=0 ; i=1,2\right)$ while input/output/feedback relations for outputs, inputs, and errors, respectively, $y_{i n}, u_{i n}$, and $e_{i n}, i=1,2$, are subject to

(1) $y_{\text {in }}=G_{i}^{n} e_{\text {in }}$ for $i=1,2$

(2) $e_{1 n}=u_{1 n}-y_{2 n}$,

$e_{2 n}=u_{2 n}+y_{1 n}$

$$
u_{i n} \in L_{2 e}^{p} \equiv L_{2 e}\left(\mathbf{R}_{0+} ; \mathbf{R}^{p}\right) \text { for } i=1,2,
$$

$\forall n \in \mathbf{Z}_{+}$. Then, the following properties hold for $i=1,2$ :

(i) (a) $G_{i}: L_{2 e}\left(\mathbf{R}_{0+} ; \mathbf{R}^{p}\right) \rightarrow L_{2 e}\left(\mathbf{R}_{0+} ; \mathbf{R}^{p}\right)$ is $\left(\left\{\bar{K}_{i n}\right\}, K_{0 i}\right.$, $\left.\bar{K}_{i}\right)$-asymptotically strongly strictly accretive with $\bar{K}_{i}=(1-$ $\left.K_{i}\right) /\left(3-K_{i}\right)$ and $\left\{\bar{K}_{i n}\right\} \rightarrow \bar{K}_{0 i}=\left(2-K_{i}-K_{0 i}\right) /\left(3-K_{i}\right)$ if $\left(I-G_{i}^{n}\right): L_{2 e}\left(\mathbf{R}_{0+} ; \mathbf{R}^{p}\right) \rightarrow L_{2 e}\left(\mathbf{R}_{0+} ; \mathbf{R}^{p}\right)$ is $\left(\left\{K_{i n}\right\}, K_{0 i}, K_{i}\right)-$ asymptotically strongly strictly pseudocontractive in the intermediate sense such that $[0, \infty) \supset\left\{K_{\text {in }}\right\} \rightarrow K_{0 i}(\in[0,1))$ and $K_{i} \in[0,1)$.

(b) $G_{i}: L_{2 e}\left(\mathbf{R}_{0+} ; \mathbf{R}^{p}\right) \rightarrow L_{2 e}\left(\mathbf{R}_{0+} ; \mathbf{R}^{p}\right)$ is $\left(\left\{\bar{K}_{i n}\right\}, \bar{K}_{i}\right)-$ asymptotically strongly strictly accretive in the intermediate sense with $\bar{K}_{i}=\left(1-K_{i}\right) /\left(3-K_{i}\right)$ and $\left\{\bar{K}_{i n}\right\} \rightarrow \bar{K}_{0 i}=$ $\left(1-K_{i}\right) /\left(3-K_{i}\right)$ for $i=1,2$ if $\left(I-G_{i}^{n}\right): L_{2 e}\left(\mathbf{R}_{0+} ; \mathbf{R}^{p}\right) \rightarrow$ $L_{2 e}\left(\mathbf{R}_{0+} ; \mathbf{R}^{p}\right)$ is $\left(\left\{K_{i n}\right\}, K_{0 i}, K_{i}\right)$-asymptotically strictly pseudocontractive in the intermediate sense such that $[0, \infty)>$ $\left\{K_{\text {in }}\right\} \rightarrow 1$ and $K_{i} \in[0,1)$.

(c) $G_{i}: L_{2 e}\left(\mathbf{R}_{0+} ; \mathbf{R}^{p}\right) \rightarrow L_{2 e}\left(\mathbf{R}_{0+} ; \mathbf{R}^{p}\right)$ is $\left(\left\{\bar{K}_{i n}\right\}, \bar{K}_{0 i}\right)-$ asymptotically strictly accretive in the intermediate sense with $\left\{\bar{K}_{i n}\right\} \rightarrow \bar{K}_{0 i}=\left(1-K_{0 i}\right) / 2$ for $i=1,2$ if $\left(I-G_{i}^{n}\right): L_{2 e}\left(\mathbf{R}_{0+} ;\right.$ $\left.\mathbf{R}^{p}\right) \rightarrow L_{2 e}\left(\mathbf{R}_{0+} ; \mathbf{R}^{p}\right)$ is $\left(\left\{K_{i n}\right\}, K_{0 i}\right)$-asymptotically strongly pseudocontractive in the intermediate sense such that $[0, \infty) \supset$ $\left\{K_{\text {in }}\right\} \rightarrow K_{0 i} \in[0,1)$.

(ii) $G_{i}: L_{2 e}\left(\mathbf{R}_{0+} ; \mathbf{R}^{p}\right) \rightarrow L_{2 e}\left(\mathbf{R}_{0+} ; \mathbf{R}^{p}\right), i=1,2$, is asymptotically incrementally strictly passive (and asymptotically strictly passive, asymptotically incrementally strictly positive, and asymptotically strictly positive) in the intermediate sense.

(iii) The binary relation $R_{G_{1}^{n} G_{2}^{n}}(u, y)=\left\{\left(\left(u_{1 n}, u_{2 n}\right),\left(y_{1 n}\right.\right.\right.$, $\left.\left.\left.y_{2 n}\right)\right): u_{1,2} \in L_{2 e}^{p}\right\}$ defined by (47)-(48) is $L_{2}^{p}$-stable $w b$.

(iv) $R_{G_{1}^{n} G_{2}^{n}}(u, y)=\left\{\left(\left(u_{1 n}, u_{2 n}\right),\left(y_{1 n}, y_{2 n}\right)\right): u_{1,2} \in L_{2 e}^{p}\right\}$ is $L_{2}^{p}$-stable $w b$ if one of the operators $\left(I-G_{i}\right)$ for some $i \in$ $\{1,2\}$ is asymptotically strongly (or strict or strict strongly) pseudocontractive in the intermediate sense and bounded while $\left(I-G_{j}\right) j(\neq i) \in\{1,2\}$ is (at least) asymptotically pseudocontractive in the intermediate sense.
Proof. From Theorem 14(v), one has via the replacements (I$\left.T^{n}\right) \rightarrow G_{i}^{n}$ for $i=1,2$

$$
\begin{aligned}
\liminf _{n \rightarrow \infty}\left(\left\langle G_{i}^{n} u_{i n}-G_{i}^{n} \bar{u}_{i n}, u_{i n}-\bar{u}_{i n}\right\rangle_{T}\right. \\
-\frac{2-K_{i}-K_{i n}}{3-K_{i}}\left\|u_{i n}-\bar{u}_{n i}\right\|_{T 2}^{2} \\
\left.\quad-\frac{1-K_{i}}{3-K_{i}}\left\|\left(I-G_{i}^{n}\right) u_{i n}-\left(I-G_{i}^{n}\right) \bar{u}_{i n}\right\|_{T 2}^{2}\right) \geq 0 ;
\end{aligned}
$$

$\forall T>0$,

$\forall y_{i n}, \bar{y}_{i n} \in D\left(G_{i}\right)$ for $i=1,2$. Since $\left\{\bar{K}_{i n}\right\} \rightarrow \bar{K}_{i 0}=(2-$ $\left.K_{i}-K_{i 0}\right) /\left(3-K_{i}\right)$ with $K_{i 0}=1$ in the strict case and $K_{i 0} \in$ $[0,1) ; i=1,2$ in the strong strict case and $0<K_{i}<1, i=1,2$, leading to $0<K_{i}+K_{i 0}<2$ for $i=1,2$ one has from (54) for all integer $n\left(\geq n_{0}\right)$ and some $n_{0} \in \mathbf{Z}_{+}$, by taking $\bar{u}_{i}=0$ for $i=1,2$, that

$$
\begin{aligned}
& \left\langle u_{i n}, G_{i}^{n} u_{i n}\right\rangle_{T}=\left\langle G_{i}^{n} u_{i}, u_{i}\right\rangle_{T} \\
& \geq \varepsilon_{\text {in }}\left\|u_{i n}\right\|_{T 2}^{2}+\varepsilon_{i}\left\|\left(I-G_{i}^{n}\right) u_{i n}\right\|_{T 2}^{2} \\
& \geq \varepsilon_{\text {in }}\left\|u_{\text {in }}\right\|_{T 2}^{2}+\varepsilon_{i} \lambda_{\text {in }}\left\|G_{i}^{n} u_{\text {in }}\right\|_{T 2}^{2} ;
\end{aligned}
$$

for $n \geq n_{0}$, where $\varepsilon_{i}=\left(1-K_{i}\right) /\left(3-K_{i}\right)>0,\left\{\varepsilon_{i n}\right\}_{n \geq n_{0}}>0$, and $\left\{\lambda_{i n}\right\}_{n \geq n_{0}}>0$ with $\varepsilon_{i n}=\left(2-K_{i}-K_{i n}\right) /\left(3-K_{i}\right)$ and $\lambda_{\text {in }}=\mu\left(I-G_{i}^{n}\right) / \mu\left(G_{i}^{n}\right)>0, n \geq n_{0}$, so that $\delta_{\text {in }}=\varepsilon_{\text {in }} \lambda_{\text {in }}, n \geq$ $n_{0}$ for $i=1,2$, since $\left(I-G_{i}^{n}\right): L_{2 e}\left(\mathbf{R}_{+} ; \mathbf{R}^{p}\right) \rightarrow L_{2 e}\left(\mathbf{R}_{+} ; \mathbf{R}^{p}\right)$ are one-to-one and of closed range, equivalently, invertible so that $\mu\left(I-G_{i}^{n}\right)>0,[10]$, for $i=1,2$ and all $n \geq n_{0}$. Property (i) follows from (54)-(55). Direct calculations with (50) subject to the input/output/feedback constraints (a) and (b) yield

$$
\begin{aligned}
& \left\langle y_{1 n}, e_{1 n}\right\rangle_{T}+\left\langle y_{2 n}, e_{2 n}\right\rangle_{T}=\left\langle y_{1 n}, u_{1 n}\right\rangle_{T}+\left\langle y_{2 n}, u_{2 n}\right\rangle_{T} \\
& \quad=\left\langle G_{1}^{n} u_{1 n}, e_{1 n}\right\rangle_{T}+\left\langle G_{2}^{n} u_{2 n}, e_{2 n}\right\rangle_{T} \\
& \quad=\left\langle G_{1}^{n} u_{1 n}, u_{1 n}\right\rangle_{T}+\left\langle G_{2}^{n} u_{2 n}, u_{2 n}\right\rangle_{T} \\
& \quad \geq \sum_{i=1}^{2}\left(\varepsilon_{i n}\left\|e_{i n}\right\|_{T 2}^{2}+\delta_{i n}\left\|G_{i}^{n} u_{i n}\right\|_{T 2}^{2}\right)+\varepsilon_{n}
\end{aligned}
$$

for some sequence $\left\{\varepsilon_{n}\right\} \subset \mathbf{R}_{+}$, with $\varepsilon_{n}=\varepsilon_{n}(T) \rightarrow 0$ as $n \rightarrow \infty$, $\forall T>0$, so that

$$
\begin{aligned}
& \liminf _{n \rightarrow \infty}\left(\left\langle y_{1 n}, e_{1 n}\right\rangle_{T}+\left\langle y_{2 n}, e_{2 n}\right\rangle_{T}\right. \\
& \left.-\sum_{i=1}^{2}\left(\varepsilon_{i n}\left\|e_{i n}\right\|_{T 2}^{2}+\delta_{i n}\left\|G_{i}^{n} u_{i n}\right\|_{T 2}^{2}\right)\right) \geq 0 .
\end{aligned}
$$

It follows from (57) after some routine calculations as those given in [1] that $R_{G_{1}^{n} G_{2}^{n}}(u, y)$ is $L_{2}^{p}$-stable wb for $n \geq n_{0}$ and asymptotically $L_{2}^{p}$-stable wb since $y_{1}, y_{2} \in L_{2}^{p}$ for any given inputs $u_{1}, u_{2} \in L_{2 e}^{p}$; thus Property (ii) follows since, 
from Property (i), $\liminf _{n \rightarrow \infty}\left(\varepsilon_{i n}+\delta_{j n}\right)>0$ for $j, i(\neq j) \epsilon$ $\{1,2\}$. Property (ii) is direct from the relations of accretivity and passivity (Theorem 14). Property (iii) follows since the condition $\lim \inf _{n \rightarrow \infty}\left(\varepsilon_{i n}+\delta_{j n}\right)>0$ for $j, i(\neq j) \in\{1,2\}$ still holds if

1. at least one of the operators $\left(I-G_{i}\right)$ on $L_{2 e}^{p} \equiv$ $L_{2 e}\left(\mathbf{R}_{+} ; \mathbf{R}^{p}\right)$ for some $i=1,2$ is bounded (then $L_{2}^{p}-$ stable wb [1]) and (at least) asymptotically strongly pseudocontractive in the intermediate sense, leading to (at least) asymptotic strict accretivity (then implying the strict passivity) in the intermediate sense of the corresponding $G_{i}$,

2. the other operator $\left(I-G_{j}\right)$ is (at least) asymptotically pseudocontractive in the intermediate sense (see Definition 12(d)-(h) and Theorems 14(v)-(vii) leading to accretivity (then implying the passivity) in the intermediate sense of the corresponding $G_{j}$.

Configuration (53) of strict passive bounded operator with a passive one is sufficient to guarantee the $L_{2}^{p}$-closed-loop stability [1] so the parallel conditions in the intermediate sense also guarantee the stability property.

Remark 23. While the operators $G_{i}$ on $L_{2 e}\left(\mathbf{R}_{0+} ; \mathbf{R}^{p}\right), G_{i}^{0}=$ $I$, for $i=1,2$, are asymptotically strictly passive (resp., passive) according to Theorem 22, the corresponding ones being asymptotically pseudocontractive in the intermediate sense or, simply, asymptotically pseudocontractive (resp., passive) are $T_{i}=I-G_{i}$ for $i=1,2$ which satisfy the recursive relations:

$$
\begin{aligned}
T_{i}^{n} & =I-G_{i}^{n}, \\
T^{0} & =0 \\
T_{i}^{n+1} & =I-G_{i}^{n+1}=I-G_{i}^{n}(I-T) \\
& =I-\left(I-T_{i}^{n}\right)(I-T)
\end{aligned}
$$

for $i=1,2, \forall n \in \mathbf{Z}_{+}$. Note that Theorem 22(iii) includes the case when both operators $\left(I-G_{i}\right)$ on $L_{2 e}\left(\mathbf{R}_{0+} ; \mathbf{R}^{p}\right)$ for $i=1,2$ are asymptotically strictly pseudocontractive. Note also that since the binary relation $R_{G_{1}^{n} G_{2}^{n}}(u, e)=\left\{\left(u_{1 n}, u_{2 n}\right),\left(e_{1 n}, e_{2 n}\right)\right.$ : $\left.u_{1,2} \in L_{2 e}^{p}\right\}$ is asymptotically $L_{2}^{p}$-stable wb under the conditions of Theorem 22(i) and under the weaker conditions of Theorem 22(iii) then the input-to-error binary $R_{u e}=$ $\left\{\left(\left(u_{1 n}, u_{2 n}\right),\left(e_{1 n}, u_{2 n}\right)\right): u_{1,2} \in L_{2 e}^{p}\right\}$.

The following result is of interest relating the convergence properties of the operators $(I-G)$ and $G$ while also relating the potential fixed points of $(I-G)$ to the convergence properties of sequences generated through the operator $G$.

Theorem 24. The following properties hold:

(i) If $C$ is a nonempty closed convex subset of a real Hilbert space $H$ and $T: C \rightarrow C$ is uniformly L-Lipschitz and asymptotically pseudocontractive in the intermediate sense then the set $\operatorname{Fix}(T)$ of fixed points of $T$ is a closed convex subset of $H$. If, furthermore, $\operatorname{Fix}(T) \neq \varnothing$ then $(I-T)$ is demiclosed at zero (Lemmas 1.4 and 1.5 of Kim et al. [18]). (ii) Assume that $(I-G): C \rightarrow C$ is uniformly L-Lipschitz and asymptotically pseudocontractive in the intermediate sense, where $C$ is a nonempty closed convex subset of $L_{2}^{p} \equiv L_{2}\left(\mathbf{R}_{0+} ; \mathbf{R}^{p}\right)$ and that $L_{2}^{p}$ is endowed with the usual inner product. Then one has the following:

1. $\operatorname{Fix}(I-G)$ is a closed convex subset of $L_{2}^{p}$.

2. If $\operatorname{Fix}(I-G) \neq \varnothing$ then $G$ is demiclosed at zero.

3. Assume that $\operatorname{Fix}(I-G) \neq \varnothing$, that $0 \in C$, and that $\left\{G^{n}\right\} \rightarrow G^{*}$ (i.e., $\left\{G^{n}\right\}$ converges pointwise to $\left.G^{*}\right)$. Thus, if $\left\{x_{n}\right\}(\subset C) \underset{w}{\rightarrow} x^{*} \in C$ and $\left\{G^{n} x_{n}\right\}(\subset C) \rightarrow 0$ (i.e., if $\left\{x_{n}\right\}$ converges weakly to $x^{*}$, namely, $\left\{\left\langle x_{n}, y\right\rangle\right\} \rightarrow$ $\left\langle x^{*}, y\right\rangle, \forall y \in C$, and $\left\{G^{n} x_{n}\right\}$ converges strongly to $0 \in C)$ then $G^{*} x^{*} \rightarrow 0$. If, in addition, $G^{*} 0=0$ and $G^{*}$ is injective at zero then $x^{*}=0$ so that $\left\{\left\langle x_{n}, y\right\rangle\right\} \rightarrow 0$, $\forall y \in C$ as a result.

(iii) Property (ii) also holds if $(I-G)$ is replaced with a sequence of L-Lipschitz and asymptotically pseudocontractive in the intermediate sense operators $\left\{I-G_{n}\right\}: C \rightarrow C$ such that $C$ is a nonempty closed convex subset of $L_{2}^{p}$, with $0 \in C$ and $\left\{G_{n}\right\} \rightarrow G^{*}$.

Proof. Since $(I-G): C \rightarrow C$ is uniformly $L$-Lipschitz and asymptotically pseudocontractive in the intermediate sense, $C$ is a nonempty closed convex subset of $L_{2}^{p}, \operatorname{Fix}(I-G) \neq$ $\varnothing$ [then $G$ is demiclosed at 0 [21] from Property (i)], $0 \in$ $C,\left\{G^{n}\right\} \rightarrow G^{*},\left\{x_{n}\right\} \underset{w}{\rightarrow} x^{*}$, and $\left\{G^{n} x_{n}\right\} \rightarrow 0$, then one has that

$$
\begin{gathered}
{\left[\left\{G^{n} x_{n}-G^{*} x_{n}\right\} \longrightarrow 0\right] \Longrightarrow} \\
{\left[\left\{G^{*} x_{n}\right\} \longrightarrow 0\right] \Longrightarrow} \\
{\left[G^{*} x^{*}=0\right] .}
\end{gathered}
$$

If, furthermore, $G^{*} 0=0$ and $G^{*}$ is injective at zero then $x^{*}=0$ since $\left[\left(G^{*} x^{*}=0\right) \wedge\left(G^{*} 0=0\right)\right] \Rightarrow\left(x^{*}=0\right)$. Hence, Property (ii) follows.

One gets directly from Theorem 24 in view of Proposition 13 the following result.

Corollary 25. Theorem 24 holds for $T: C \rightarrow C$ being uniformly L-Lipschitz and asymptotically strictly, or strongly strictly, pseudocontractive in the intermediate sense.

Remark 26. Theorem 22 is applicable to asymptotic passivity and incremental asymptotic passivity of, in general, a nonlinear dynamic system described by two operators connected in feedback form, one of them describing the controlled object while the other one describes the feedback controller. The passivity conditions are guaranteed if two associated related operators are, respectively, asymptotically strictly pseudocontractive and/or asymptotically strictly pseudocontractive in the intermediate sense. The related discussion follows below.

Consider the nonlinear control system [12]:

$$
\begin{aligned}
\dot{x}(t) & =f(x)+L(x) u, \\
y & =G(x),
\end{aligned}
$$


where $x=x(t) \in \mathbf{R}^{n}, u=u(t) \in \mathbf{R}^{m}$, and $y=y(t) \in \mathbf{R}^{\ell}$ are the state, input, and output vectors and, respectively, $f$ and $G$ are smooth vector-valued functions on $x$, and $L$ is a smooth matrix-valued function on $x$.

Definition 27 (see [12]). System (60) is called strictly $S$ passive (resp., $S$-passive) if there exists a nonnegative scalar function (storage function) $V(x)$ and a scalar function $\mu(x)$, where $\mu(x)>0$ for $x \neq 0$, such that

$$
V(x) \leq V\left(x_{0}\right)+\int_{0}^{t}\left(u^{T}(\sigma) S y(\sigma)-\mu(x(\sigma))\right) d \sigma,
$$

respectively,

$$
V(x) \leq V\left(x_{0}\right)+\int_{0}^{t} u^{T}(\sigma) S y(\sigma) d \sigma
$$

for any $t \geq 0$ and for any solution of system (60) satisfying $x(0)=x_{0}$ and $x(t) \equiv x$, where $S$ is a prespecified $m \times \ell$ real matrix.

From Definitions 1 and 27, we have immediately the following simple direct result.

Proposition 28. Assume that $m=\ell$ and that $G: L_{2 e}\left(\mathbf{R}_{0+}\right.$; $\left.\mathbf{R}^{m}\right) \rightarrow L_{2 e}\left(\mathbf{R}_{0+} ; \mathbf{R}^{m}\right)$ in (60). Then one has the following:

1. If $G: L_{2 e}\left(\mathbf{R}_{0+} ; \mathbf{R}^{m}\right) \rightarrow L_{2 e}\left(\mathbf{R}_{0+} ; \mathbf{R}^{m}\right)$ is passive (resp., strictly passive) then the system (60) is $I_{m}$-passive (resp., strictly $I_{m}$-passive) and conversely.

2. If $G: L_{2 e}\left(\mathbf{R}_{0+} ; \mathbf{R}^{m}\right) \rightarrow L_{2 e}\left(\mathbf{R}_{0+} ; \mathbf{R}^{m}\right)$ is strictly passive or strongly strictly passive then the system (60) is $I_{m^{-}}$ strictly passive and conversely.

Proof. One has from (61) that

$$
\begin{aligned}
\langle u, y\rangle_{T} & =\langle u, G u\rangle_{T} \geq \varepsilon_{\mu}\langle u, u\rangle_{T}+V(x)-V\left(x_{0}\right) \\
& \geq \varepsilon_{\mu}\|u\|_{T 2}^{2}-\beta \geq-\beta
\end{aligned}
$$

for any $T \geq 0$ since $V(x)$ is everywhere nonnegative where $\varepsilon_{\mu}=\inf _{T \geq 0}\left(\inf _{t \geq 0}\left(\mu(t) /\|u\|_{T 2}\right)\right) \geq 0$, the inequality being strict if (60) is strictly $I_{m}$-passive, and $\varepsilon_{\mu}=0$ if (60) is $I_{m^{-}}$ passive, with $\beta=V\left(x_{0}\right) \geq 0$. Conversely, the existence of the storage function being constant, that is, $V(x)=V\left(x_{0}\right)$, proves the converse assertion; that is, if (60) is $I_{m}$-passive then the operator $G$ is passive (resp., strictly passive). Property (i) has been proved. To prove Property (ii), define the storage function $V=V(y)=V(G x)=\left(\inf _{T \geq 0}\left(\delta_{0 G} /\|y\|_{T}\right)\right)\langle y, y\rangle_{T}$ for any $T \geq 0$ and some real constant $\delta_{0 G}>0$ is taken as a nonnegative function of the output, then, from (54) for $\delta_{G}=\inf _{T \geq 0}\left(\delta_{0 G} /\|y\|_{T}\right)$,

$$
\langle u, G u\rangle_{T} \geq \varepsilon_{\mu}\|u\|_{T 2}^{2}+\delta_{G}\|y\|_{T 2}^{2}-\beta
$$

for any real $T \geq 0$ with $\varepsilon_{\mu}+\delta_{G}>0$ if $G: L_{2 e}\left(\mathbf{R}_{+} ; \mathbf{R}^{m}\right) \rightarrow$ $L_{2 e}\left(\mathbf{R}_{+} ; \mathbf{R}^{m}\right)$ is strongly strictly passive, hence Property (ii).
Remark 29. Inequality (64) refers to the operator $G$ being strictly passive if the sum of the two right-hand-side constants is positive. Conversely, control system (60) is $I_{m}$-strictly passive. Borrowing the terminology of [13], if $\varepsilon_{\mu}>0$, then system (60) is said to be strictly input passive while if $\delta_{G}>0$, then system (52) is said to be strictly output passive.

If both right-hand side constants of (64) are positive, then the operator $G$ is, furthermore, strongly strictly passive.

The last right-hand-side inequality of (63), that is, $\langle u, y\rangle_{T} \geq-\beta$, is commonly referred to as Popov's inequality $[3-5,12,22]$ which is a basic tool to characterize the hyperstability and asymptotic hyperstability of feedback systems where the feed-forward loop is a passive linear dynamic system while the nonlinear feedback controller belongs to a general class satisfying such a passivity-type constraint. A well-known related result is as follows.

Theorem 30. The following properties hold.

(i) Assume that

1. $y=G u$ with $u$ and $y$ being the input and output signals of dimension $p \geq 1$ in $L_{2 e}^{p}$ and $G$ being described in the frequency domain by a strictly positive real transfer matrix $\widehat{G}(s)$,

2. $u=-K y$ with $K \in K_{\Omega}$ being a feedback nonlinear and perhaps time-varying controller causal operator for the controlled system described by $G$ satisfying the inequality $\langle y, K y\rangle_{T} \geq-\beta ; \forall K \in K_{\Omega}$, for any arbitrary real constant $\beta>0$.

Then, the closed-loop controlled system is globally asymptotically Lyapunov's stable for any controller $K$ belonging to the set $K_{\Omega}$; that is, it is asymptotically hyperstable for the class $K_{\Omega}$; thus $u$ and $y$ are bounded square-integrable functions on $[0, \infty)$ (i.e., in $L_{2 e}^{p}$ ) and $u(t) \rightarrow 0$ and $y(t) \rightarrow 0$ as $t \rightarrow \infty$ for any given bounded initial conditions of the state.

(ii) Assume that $y=G u$ and $u=-K y$, with $u$ and $y$ being the input and output signals of dimension $p \geq 1$ in $L_{2 e}^{p}$. Then, the closed-loop system is globally asymptotically Lyapunov's stable if $G$ is strictly input passive and bounded and $K$ is passive with zero-independent constant or if $K$ is strictly input passive and bounded and $G$ is passive with zero-independent constant.

Proof. Since $G(s)$ is a positive real transfer matrix then it is a bounded self-adjoint causal operator which is strictly stable with strictly positive real, that is, $\operatorname{Re}(G(s)) \geq \beta_{1}>0$ for all $s \in \mathrm{C}$ with $\operatorname{Re} s \geq 0$. Thus, one has, for any real constant $\beta \in\left(\beta_{1}, \infty\right)$ and some real constant $\beta_{1}>0$ and for any given control $L_{2 e}^{p}$ with support of nonzero measure,

$$
\begin{aligned}
\infty & >\beta \geq-\langle K y, y\rangle_{T}=-\left\langle(K y)_{T}, y_{T}\right\rangle \\
& =-\left\langle(K y)_{T}, y_{T}\right\rangle_{T}=-\left\langle(K y)_{T}, y_{T}\right\rangle_{T} \\
& =-\left\langle K y, y_{T}\right\rangle_{T}=\langle y, u\rangle_{T}=\langle G u, u\rangle_{T} \\
& =\left\langle(G u)_{T}, u_{T}\right\rangle=\left\langle(G u)_{T}, u_{T}\right\rangle_{T}=\left\langle(G u)_{T}, u\right\rangle_{T} \\
& =\left\langle G u, u_{T}\right\rangle_{T}=\frac{1}{2 \pi} \int_{-\infty}^{\infty} \widehat{u}_{T}^{T}(-\mathbf{i} \omega) \widehat{G}(\mathbf{i} \omega) \widehat{u}_{T}(\mathbf{i} \omega) d \omega
\end{aligned}
$$




$$
\begin{aligned}
& \geq \frac{1}{4 \pi} \min _{\omega \in \mathbf{R}_{0+}}\left(\lambda_{\min }\left[\widehat{G}(\mathbf{i} \omega)+\widehat{G}^{T}(-\mathbf{i} \omega)\right]\right) \\
& \cdot \int_{-\infty}^{\infty} \widehat{u}_{T}^{T}(-\mathbf{i} \omega) \widehat{u}_{T}(\mathbf{i} \omega) d \omega \geq \frac{\beta_{1}}{4 \pi}\|u\|_{T 2}^{2}>0 ;
\end{aligned}
$$

$\forall T>0$,

where $\lambda_{\min }(\cdot)$ denotes the minimum eigenvalue of the symmetric (.)-matrix, so that the controlled system $y=G u$ is input-strictly passive, and the operator $G$ is strictly positive, strictly passive, and strictly accretive. Thus, the Fourier transform $\widehat{u}_{T}$ of $u_{T}$ exists since $u \in L_{2 e}$ and the Laplace transform $\widehat{G}(s)$ and the associated Fourier transform $\widehat{G}(\mathbf{i} \omega)=$ $\widehat{G}^{T}(-\mathbf{i} \omega)$ of $G$ exist as well for any complex number $s=$ $\sigma+\mathbf{i} \omega$ with $\sigma \in \mathbf{R}_{+}$and $\omega \in \mathbf{R}_{0+}$ since $\widehat{G}$ is strictly positive real, then analytic for $\sigma=\operatorname{Re} s \geq 0$ and subject to $\lambda_{\text {min }}\left[\widehat{G}(\mathbf{i} \omega)+\widehat{G}^{T}(-\mathbf{i} \omega)\right] \geq \beta_{1}>0, \forall \omega \in \mathbf{R}_{0+}$, where $\lambda_{\text {min }}(\cdot)$ denotes the minimum eigenvalue and the superscript " $T$ " denotes transpose. Thus, Parseval's theorem can be applied in (65). Furthermore, the controller operator $K$ is passive since $\langle y, K y\rangle_{T} \geq-\beta ; \forall T>0$, since $\beta \geq 0$ is arbitrary, $u$ and $y$ are bounded square-integrable functions on $[0, \infty)$ (i.e., in $L_{2 e}^{p}$ ) and $u_{T}(t) \rightarrow 0$ and $y_{T}(t) \rightarrow 0$ as $t \rightarrow \infty$ so that $u(t) \rightarrow 0$ and $y(t) \rightarrow 0$ as $t \rightarrow \infty$ for any bounded initial conditions. Property (i) has been proved.

To prove Property (ii), note that, in this case, if $\gamma_{2}(G)$ and $\gamma_{2}(K)$ are the $L_{2}$-gains wb of $G$ and $K$, then one has for any control being nonzero except possibly on a set of zero measure that

$$
\begin{aligned}
+\infty & >\min \left(\beta_{y}-\varepsilon_{y}\|y\|_{T 2}^{2}, \gamma_{2}(G)\|u\|_{T 2}^{2}\right) \\
& \geq-\langle y, K y\rangle_{T}=\langle y, u\rangle_{T}=\langle G u, u\rangle_{T} \\
& \geq-\beta_{u}+\varepsilon_{u}\|u\|_{T 2}^{2} \geq \gamma_{1} \gamma_{2}(G)\|u\|_{T 2}^{2}>0 ;
\end{aligned}
$$

$\forall T>0$,

which holds provided that $\gamma_{1} \in\left(0, \varepsilon_{u} / \gamma_{2}(G)\right], \gamma_{2}(G)<+\infty$, $\beta_{u}=\varepsilon_{y}=0$, and $\beta_{y} \geq 0, \varepsilon_{u}>0$; that is, $G$ is bounded and strictly input passive and $K$ is passive, or

$$
\begin{aligned}
0 & <\gamma_{2} \gamma_{2}(K)\|y\|_{T 2}^{2} \leq-\beta_{y}+\varepsilon_{y}\|y\|_{T 2}^{2} \leq\langle y, K y\rangle_{T} \\
& =-\langle y, u\rangle_{T}=-\langle G u, u\rangle_{T} \\
& \leq \min \left(\beta_{u}-\varepsilon_{u}\|u\|_{T 2}^{2}, \gamma_{2}(K)\|y\|_{T 2}^{2}\right)<+\infty
\end{aligned}
$$

$\forall T>0$,

which holds provided that $\gamma_{2} \in\left(0, \varepsilon_{y} / \gamma_{2}(K)\right], \gamma_{2}(K)<+\infty$, $\beta_{y}=\varepsilon_{u}=0, \beta_{u} \geq 0$, and $\varepsilon_{y}>0$; that is, $K$ is bounded and strictly input passive and $G$ is passive. On the other hand if $G$ is bounded and strictly input passive and $K$ is passive with $\beta_{y 0}=0$, then it is also passive with any finite positive constant $\beta_{y}$ so that one gets from $(66)$ that $u_{T}(t) \rightarrow 0$ and $y_{T}(t) \rightarrow 0$ as $t \rightarrow \infty$ for any $T>0$ and then $u(t) \rightarrow 0$ and $y(t) \rightarrow 0$ as $t \rightarrow \infty$, for any given initial conditions. A similar conclusion arises from (67) if $K$ is bounded and strictly input passive and $G$ is passive with $\beta_{u 0}=0$. Property (ii) has been proved.
Theorem 30 can be directly reformulated in the discretetime framework related to the space $\ell_{2 e}^{p}$.

\section{Further Examples}

Example 1. Consider the iterated linear continuous-time dynamic feedback system:

(a) $e_{1 n}=u_{1 n}-y_{2 n}$,

$$
\begin{aligned}
& e_{2 n}=u_{2 n}+y_{1 n}, \\
& u_{i n} \in L_{2 e}^{p} \equiv L_{2 e}\left(\mathbf{R}_{0+} ; \mathbf{R}^{p}\right) \text { for } i=1,2 ; \forall n \in \mathbf{Z}_{+} \\
& \text {(b) } y_{1 n}=\left(I-G_{1}^{n}\right) e_{1 n}=\left(I-G_{1}^{n}\right)\left(u_{1 n}-y_{2 n}\right) ;
\end{aligned}
$$$$
\forall n \in \mathbf{Z}_{+}
$$

$$
\begin{array}{r}
y_{2 n}=\left(I-G_{2}^{n}\right) e_{2 n}=\left(I-G_{2}^{n}\right)\left(u_{2 n}+y_{1 n}\right) ; \\
\forall n \in \mathbf{Z}_{+},
\end{array}
$$

for given initial conditions $y_{i 0}=a_{i}$ for $i=1,2$, which is described by the sequences of operators $\left\{\left(I-G_{i}^{n}\right)\right\}$ on $L_{2 e}^{p}$. Then, if $G_{i}^{n}$ are linear for $i=1,2$,

$$
\begin{aligned}
y_{1 n} & =\left(I-G_{1}^{n}\right) u_{1 n}-\left(I-G_{1}^{n}\right)\left(I-G_{2}^{n}\right)\left(u_{2 n}+y_{1 n}\right) \\
& =\left[I+\left(I-G_{1}^{n}\right)\left(I-G_{2}^{n}\right)\right]^{-1} \\
\cdot & {\left[\left(I-G_{1}^{n}\right) u_{1 n}-\left(I-G_{1}^{n}\right)\left(I-G_{2}^{n}\right) u_{2 n}\right] ; }
\end{aligned}
$$

$\forall n \in \mathbf{Z}_{+}$,

$$
\begin{aligned}
y_{2 n} & =\left(I-G_{2}^{n}\right)\left(u_{2 n}+y_{1 n}\right)=\left(I-G_{2}^{n}\right) u_{2 n} \\
- & \left(I-G_{2}^{n}\right)\left(I-G_{1}^{n}\right)\left(u_{1 n}+y_{2 n}\right) \\
= & {\left[I+\left(I-G_{2}^{n}\right)\left(I-G_{1}^{n}\right)\right]^{-1} } \\
\cdot & {\left[\left(I-G_{2}^{n}\right) u_{2 n}-\left(I-G_{2}^{n}\right)\left(I-G_{1}^{n}\right) u_{1 n}\right] ; }
\end{aligned}
$$

$\forall n \in \mathbf{Z}_{+}$

provided that the given inverses exists. Then (68) and (69a)(69b) are well-posed in the sense that for each control input $u_{n}=\left(u_{1 n}^{T}, u_{2 n}^{T}\right) \in L_{2 e}^{2 p}$ the output $y_{n}=\left(y_{1 n}^{T}, y_{2 n}^{T}\right) \in L_{2 e}^{2 p}$ and so the input-output error $e_{n}=\left(e_{1 n}^{T}, e_{2 n}^{T}\right) \in L_{2 e}^{2 p}$ exist and are unique and, equivalently, the operators $\left[I+\left(I-G_{i}^{n}\right)\left(I-G_{j}^{n}\right)\right]^{-1}$ on $L_{2 e}^{p}$ for $i, j(\neq i) \in\{1,2\}$ are causal and then well-posed and then (67) holds. Note that a mapping $A: L_{q e}^{p} \rightarrow L_{q e}^{p}$ for $q \in[1, \infty)$ is causal if $(A f)_{T}=\left(A f_{T}\right)_{T}, \forall T \geq 0, \forall f \in L_{q e}^{p}$, $[1,13]$. Note also that the particular case that the inputs $u_{1}$ and $u_{2}$ are independent of $n$ is included.

On the other hand, if $G_{i}$ is asymptotically pseudocontractive in the intermediate sense for some $i \in\{1,2\}$ and $G_{j}$ is asymptotically strictly (or strongly strictly) pseudocontractive in the intermediate sense for $j(\neq i) \in\{1,2\}$ then $\left(I-G_{i}\right)$ are asymptotically strictly passive and incrementally asymptotically passive for $i=1,2$ and the binary relation $R_{\left(I-G_{1}^{n}\right)\left(I-G_{2}^{n}\right)}$ is asymptotically $L_{2}^{p}$-stable wb (Theorem 22 ). As 
a result, if $u_{1,2}$ are square-integrable, then $e_{1,2}$ and $y_{1,2}$ are also square-integrable and converge asymptotically to zero except possibly on a set of zero measures.

A dual problem to the above one is as follows. If $\left(I-G_{i}\right)$ is either asymptotically strictly or strongly strictly pseudocontractive in the intermediate sense for some $i \in\{1,2\}$ then $G_{i}$ is asymptotically strictly positive and passive (if self-adjoint), asymptotically strongly strictly accretive, and incrementally asymptotically passive (see Remark 15).

Example 2. If the above system is not linear (i.e., both $G_{1}, G_{2}$ are not jointly linear) then (70a) and (70b) do not necessarily hold. However, the following equations hold also if the operators are nonlinear [1]:

$$
\begin{aligned}
e & =u-F y \\
y & =(I-G) e \\
& =(I-G)(u-F(I-G)) e \Longleftrightarrow \\
(I+F(I-G)) e & =u \\
y & =(I-G)(u-F y) \Longleftrightarrow \\
(I+(I-G) F) y & =(I-G) u
\end{aligned}
$$

for given initial conditions $y_{i 0}=a_{i}$ for $i=1,2$, where $e=\left(e_{1}^{T}, e_{2}^{T}\right)^{T}, y=\left(y_{1}^{T}, y_{2}^{T}\right)^{T}, u=\left(u_{1}^{T}, u_{2}^{T}\right)^{T}, G=$ Block $\operatorname{Diag}\left(G_{1}, G_{2}\right), F=\left[\begin{array}{cc}0 & I_{p} \\ -I_{p} & 0\end{array}\right]$. Then, the linearity assumption of Example 1 is not necessary since the error can be described via $e=(I+F G)^{-1} u$ provided that the above inverse exists. Thus, the given results of well-posedness, passivity, and wb-stability still hold.

Remark 31. Examples 1 and 2 can be directly extended to the case that $u_{i}, e_{i}$, and $y_{i}$ have distinct dimensions $p_{i}$ for $i=1,2$ with $p_{1} \neq p_{2}$. In particular, $F=\left[\begin{array}{cc}0 & I_{p_{1}} \\ -I_{p_{2}} & 0\end{array}\right]$ in Example 2. The formalism can be also extended directly to the case $u_{i}, e_{i}, y_{i} \in$ $L_{q e}^{p_{i}}$ for $i=1,2$ and $q \in[1, \infty)$.

Example 3. Reconsider Example 1 in the discrete-time framework with the operators being nonlinear and $p_{1} \neq p_{2}$, in general. Then, $\left\{u_{i n}\right\} \in \ell_{2 e}^{p_{i}} \equiv \ell_{2 e}\left(\mathbf{Z}_{0+} ; \mathbf{R}^{p_{i}}\right)$ (i.e., the set of square summable real sequences) for $i, j=1,2$ so that (69a) and (69b) become modified as

$$
\begin{aligned}
y_{1, n+1}=\left(I-G_{1}^{n}\right) e_{1 n}=\left(I-G_{1}^{n}\right)\left(u_{1 n}-y_{2 n}\right) & \\
& \forall n \in \mathbf{Z}_{+} \\
y_{2, n+1}=\left(I-G_{2}^{n}\right) e_{2 n}=\left(I-G_{2}^{n}\right)\left(u_{2 n}+y_{1 n}\right) ; & \\
& \forall n \in \mathbf{Z}_{+} .
\end{aligned}
$$

The problem is assumed to be well-posed then $\left\{u_{i n}\right\} \in \ell_{2 e}^{p_{i}} \equiv$ $\ell_{2 e}\left(\mathbf{Z}_{0+} ; \mathbf{R}^{p_{i}}\right) ; i, j=1,2$ implies that $\left\{y_{i n}\right\},\left\{e_{i n}\right\} \in \ell_{2 e}^{p_{i}} \equiv$ $\ell_{2 e}\left(\mathbf{Z}_{0+} ; \mathbf{R}^{p_{i}}\right) ; i, j=1,2$. Assume that

$$
\begin{gathered}
{\left[\left(u_{1 n_{0}}=0\right) \wedge\left(u_{2 n_{0}}=0\right), n_{0} \in \mathbf{Z}_{0+}\right] \Longrightarrow} \\
{\left[\left(u_{1 n_{0}}=0\right) \wedge\left(u_{2 n_{0}}=0\right), n\left(\geq n_{0}\right) \in \mathbf{Z}_{0+}\right]}
\end{gathered}
$$

that is, if both inputs are zero in finite discrete-time $n_{0}$ then $\left\{u_{\text {in }}\right\}_{n \geq n_{0}} \equiv 0$,

$$
u_{i, n+1}=K_{i 1 n} u_{1 n}+K_{i 2 n} u_{2 n} ; \quad i, j=1,2, \forall n \in \mathbf{Z}_{+},
$$

for some no-necessarily unique, gain sequences $\left\{K_{i j n}\right\}, i, j=$ 1,2 , Note that, since, from the previous assumption, if both input sequences are simultaneously zero in finite time, then they become identically zero afterwards, any input sequences satisfying such a constraint can be described in this way. Consider the discrete extended dynamic system of input and output sequences $\left\{\bar{u}_{n}\right\}$ and $\left\{\bar{y}_{n}\right\}$ of dimension $p=2\left(p_{1}+\right.$ $\left.p_{2}\right)$ :

$$
\begin{aligned}
\bar{y}_{n+1}= & {\left[\begin{array}{c}
y_{1, n+1} \\
y_{2, n+1} \\
u_{1, n+1} \\
u_{2, n+1}
\end{array}\right] } \\
= & {\left[\begin{array}{cccc}
0 & G_{1}^{n}-I_{p_{1}} & I_{p_{1}}-G_{1}^{n} & 0 \\
I_{p_{2}}+G_{2}^{n} & 0 & 0 & I_{p_{2}}-G_{2}^{n} \\
0 & 0 & K_{11 n} & K_{12 n} \\
0 & 0 & K_{21 n} & K_{22 n}
\end{array}\right] \bar{u}_{n} ; } \\
&
\end{aligned}
$$

Under a compact description, one has equivalently $\bar{y}_{n+1}=$ $\left[I_{p}-\bar{G}_{n}\right] \bar{u}_{n}, \forall n \in \mathbf{Z}_{+}$, where

$$
\begin{aligned}
& \bar{G}_{n} \\
& =\left[\begin{array}{cccc}
I_{p_{1}} & I_{p_{1}}-G_{1}^{n} & G_{1}^{n}-I_{p_{1}} & 0 \\
-\left(I_{p_{2}}+G_{2}^{n}\right) & I_{p_{2}} & 0 & G_{2}^{n}-I_{p_{2}} \\
0 & 0 & I_{p_{1}}-K_{11 n} & -K_{12 n} \\
0 & 0 & -K_{21 n} & I_{p_{2}}-K_{22 n}
\end{array}\right] ; \\
& \\
&
\end{aligned}
$$

or, dually, $\bar{y}_{n+1}=G_{n} \bar{u}_{n}, \forall n \in \mathbf{Z}_{+}$, where

$$
\begin{aligned}
G_{n} & =I_{p}-\bar{G}_{n} \\
& =\left[\begin{array}{cccc}
0 & G_{1}^{n}-I_{p_{1}} & I_{p_{1}}-G_{1}^{n} & 0 \\
I_{p_{2}}+G_{2}^{n} & 0 & 0 & I_{p_{2}}-G_{2}^{n} \\
0 & 0 & K_{11 n} & K_{12 n} \\
0 & 0 & K_{21 n} & K_{22 n}
\end{array}\right] ; \\
&
\end{aligned}
$$

Thus, if $\left(I_{p}-\bar{G}\right)$, respectively $G$, is either asymptotically strictly, or strongly strictly, pseudocontractive, in the intermediate sense, then $\bar{G}$, respectively $\left(I_{p}-G\right)$, is asymptotically 
strictly passive, asymptotically strongly strictly accretive, and incrementally asymptotically passive. The eventual possible extensions of the pseudocontractive conditions related to positive realness/passivity in both continuous-time and discrete-time formalisms in dynamic systems including the eventual presence of known or unknown internal and external delays and parametrical disturbances based on previous background results [34-38] are under study.

\section{Conflicts of Interest}

The author declares that he has no conflicts of interest.

\section{Acknowledgments}

The author is very grateful to the Spanish Government and European Fund of Regional Development FEDER for Grant DPI2015-64766-R and to UPV/EHU for Grant PGC 17/33.

\section{References}

[1] M. Vidyasagar, Nonlinear Systems Analysis, Prentice Hall Inc, Englewood Cliffs, NJ, USA, 1993.

[2] B. Brogliato, R. Lozano, B. Maschke, and O. Egeland, Dissipative Systems Analysis and Control. Theory and Application, Springer, Berlin, Germany, 2007.

[3] M. de la Sen, "Stability of composite systems with an asymptotically hyperstable subsystem," International Journal of Control, vol. 44, no. 6, pp. 1769-1775, 1986.

[4] M. De la Sen, S. Alonso-Quesada, and A. Ibeas, "On the asymptotic hyperstability of switched systems under integraltype feedback regulation Popovian constraints," IMA Journal of Mathematical Control and Information, vol. 32, no. 2, pp. 359386, 2015

[5] M. De la Sen, "Asymptotic hyperstability under unstructured and structured modeling deviations from the linear behavior," Nonlinear Analysis. Real World Applications. An International Multidisciplinary Journal, vol. 7, no. 2, pp. 248-264, 2006.

[6] A. Megretski, "Lyapunov Functions and Storage Functions," in Massachusetts Institute of Technology, Lecture 5, 6.243j, 2003.

[7] I. D. Landau, Adaptive Control: The Model Reference Approach, Marcel Dekker, New York, NY, USA, 1979.

[8] V.-M. Popov, Hyperstability of Control Systems, Springer, New York, NY, USA, 1973.

[9] A. Baños and A. Barreiro, Reset control systems, Advances in Industrial Control, Springer, London, UK, 2012.

[10] A. Feintuch, Robust control theory in Hilbert space, vol. 130 of Applied Mathematical Sciences, Springer, Berlin, Germany, 1998.

[11] P. J. Moylan and D. J. Hill, "Stability Criteria for Large-Scale Systems," IEEE Transactions on Automatic Control, vol. 23, no. 2, pp. 143-149, 1978.

[12] A. L. Fradkov, "Passification of non-square linear systems and feedback Yakubovich-Kalman-Popov Lemma," European Journal of Control, vol. 9, no. 6, pp. 577-586, 2003.

[13] N. Kottenstette and P. J. Antsaklis, "Relationships between positive real, passive dissipative, \& positive systems," in Proceedings of the 2010 American Control Conference, ACC 2010, pp. 409416, July 2010.

[14] M. De la Sen, "Stability of impulsive time-varying systems and compactness of the operators mapping the input space into the state and output spaces," Journal of Mathematical Analysis and Applications, vol. 321, no. 2, pp. 621-650, 2006.

[15] W. P. Heath, J. Carrasco, and M. de la Sen, "Second-order counterexamples to the discrete-time Kalman conjecture," Automatica. A Journal of IFAC, the International Federation of Automatic Control, vol. 60, pp. 140-144, 2015.

[16] V. Berinde, "Iterative approximation of fixed points," Lecture Notes in Mathematics, vol. 1912, pp. 1-338, 2007.

[17] X. Qin and L. Wang, "On asymptotically quasi- $\varphi$-nonexpansive mappings in the intermediate sense," Abstract and Applied Analysis, vol. 2012, Article ID 636217, 13 pages, 2012.

[18] J. K. Kim, X. Qin, and S. Y. Cho, "Convergence theorems on asymptotically pseudocontractive mappings in the intermediate sense," Fixed Point Theory and Applications, vol. 2010, Article ID $186874,2010$.

[19] M. De la Sen, "On best proximity points for pseudocontractions in the intermediate sense for non-cyclic and cyclic selfmappings in metric spaces," Fixed Point Theory and Applications, 2013:146, 24 pages, 2013.

[20] M. De la Sen, "Fixed and best proximity points of cyclic jointly accretive and contractive self-mappings," Journal of Applied Mathematics, Article ID 817193, Art. ID 817193, 29 pages, 2012.

[21] A. Bnouhachem, "Strong convergence algorithm for split equilibrium problems and hierarchical fixed point problems," The Scientific World Journal, vol. 2014, Article ID 390956, 12 pages, 2014.

[22] S. S. Boiko, V. K. Dubovoj, B. Fritsche, and B. Kirstein, "Contractive operators, defect functions and scattering theory," Ukrainian Mathematical Journal, vol. 49, no. 4, pp. 481-489, 1997.

[23] D. Thakkar and R. Das, "Topological stability of a sequence of maps on a compact metric space," Bulletin of Mathematical Sciences, vol. 4, no. 1, pp. 99-111, 2014.

[24] M. Mursaleen and K. J. Ansari, "On the stability of some positive linear operators from approximation theory," Bulletin of Mathematical Sciences, vol. 5, no. 2, pp. 147-157, 2015.

[25] W. A. Kirk and N. Shahzad, "Continuation methods in certain metric and geodesic spaces," Bulletin of Mathematical Sciences, vol. 6, no. 2, pp. 311-323, 2016.

[26] M. de la Sen and A. Ibeas, "On the global asymptotic stability of switched linear time-varying systems with constant point delays," Discrete Dynamics in Nature and Society. An International Multidisciplinary Research and Review Journal, Article ID 231710, Art. ID 231710, 31 pages, 2008.

[27] S. A. Mohiuddine, J. M. Rassias, and A. Alotaibi, "Solution of the Ulam stability problem for Euler-Lagrange-Jensen $k$-quintic mappings," Mathematical Methods in the Applied Sciences, vol. 40, no. 8, pp. 3017-3025, 2017.

[28] J. Garcia-Falset and S. Reich, "Zeroes of accretive operators and the asymptotic behavior of nonlinear semigroups," Houston Journal of Mathematics, vol. 32, no. 4, pp. 1197-1225, 2006.

[29] M. de la Sen, "Preserving positive realness through discretization," Positivity. An International Journal devoted to the Theory and Applications of Positivity in Analysis, vol. 6, no. 1, pp. 31-45, 2002.

[30] C. Batlle, "Passive Control Theory. Parts I-II," in II Eurocon/ Geoplex Summer School on Modelling and Control of Complex Dynamic Systems, Bernitoro, Italy, 2005.

[31] L. Duan and C. Huang, "Existence and global attractivity of almost periodic solutions for a delayed differential neoclassical growth model," Mathematical Methods in the Applied Sciences, vol. 40, no. 3, pp. 814-822, 2017. 
[32] J.-W. Xiao, M.-J. Hu, Y.-W. Wang, and W.-H. Chen, "Impulsive positive observers and dynamic output feedback stabilization of positive linear continuous systems," International Journal of Robust and Nonlinear Control, 2016.

[33] H. Trinh, D. C. Huong, L. V. Hien, and S. Nahavandi, "Design of Reduced-Order Positive Linear Functional Observers for Positive Time-Delay Systems," IEEE Transactions on Circuits and Systems II: Express Briefs, vol. 64, no. 5, pp. 555-559, 2017.

[34] M. De la Sen, "Sufficiency-type stability and stabilization criteria for linear time-invariant systems with constant point delays," Acta Applicandae Mathematicae, vol. 83, no. 3, pp. 235256, 2004.

[35] H. J. Marquez and C. J. Damaren, "On the design of strictly positive real transfer functions," IEEE Transactions on Circuits and Systems. I. Fundamental Theory and Applications, vol. 42, no. 4, pp. 214-218, 1995.

[36] A. Znidi, K. Dehri, and A. S. Nouri, "Discrete variable structure model reference adaptive control for non strictly positive real systems using only I/O measurements," Lecture Notes in Electrical Engineering, vol. 411, pp. 98-113, 2017.

[37] A. Ferrante and L. Ntogramatzidis, "Solvability conditions for the positive real lemma equations in the discrete time," IET Control Theory Applications, 2017.

[38] A. Li, G. Liu, Y. Luo, and X. Yang, "An indirect Lyapunov approach to robust stabilization for a class of linear fractionalorder system with positive real uncertainty," Journal of Applied Mathematics and Computing, pp. 1-17, 2017. 


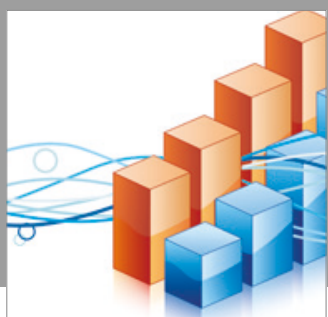

Advances in

Operations Research

vatersals

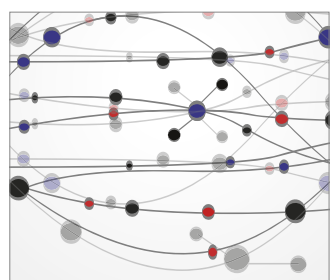

\section{The Scientific} World Journal
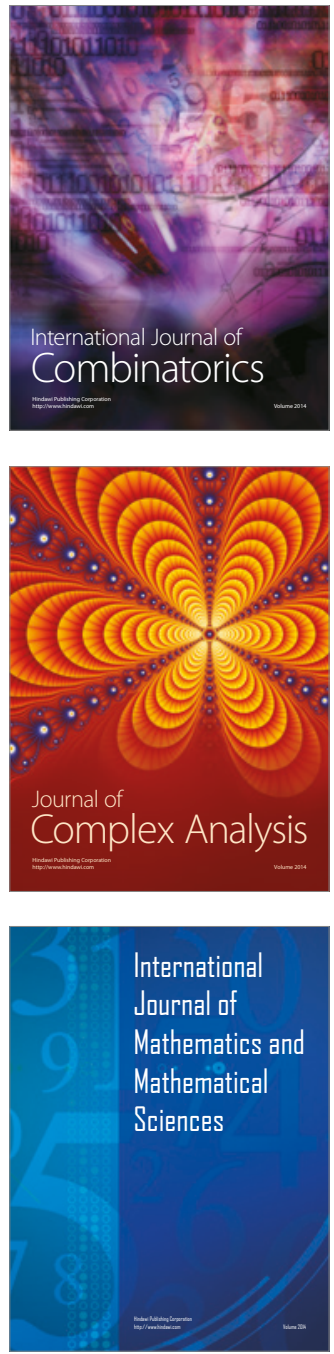
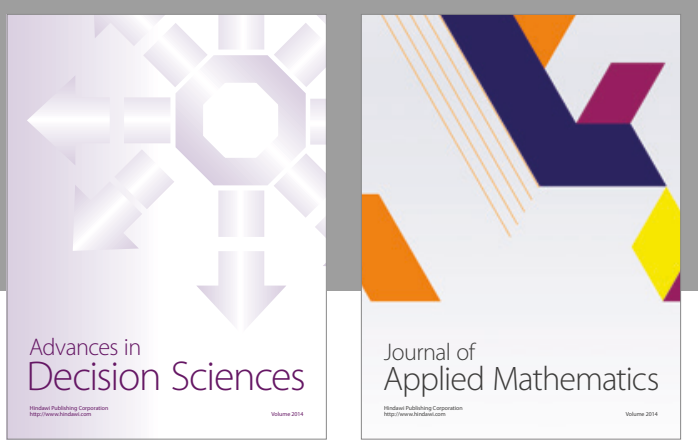

Algebra

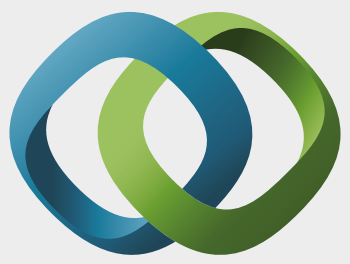

\section{Hindawi}

Submit your manuscripts at

https://www.hindawi.com
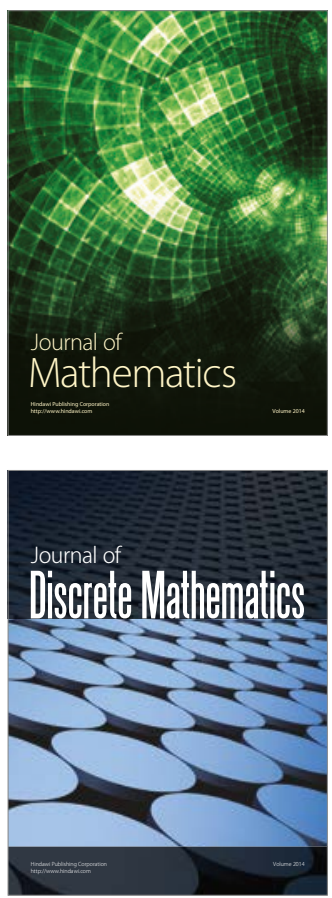

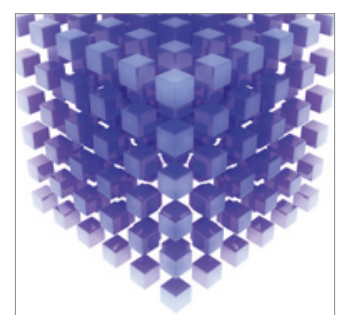

Mathematical Problems in Engineering
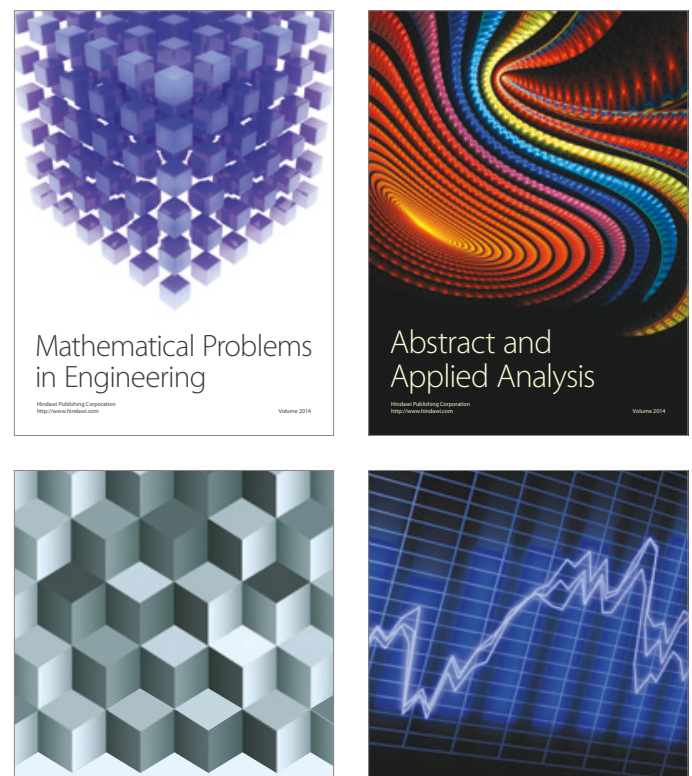

Journal of

Function Spaces

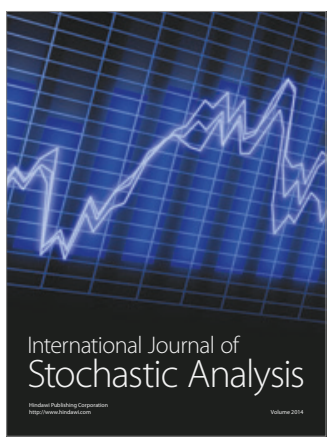

Probability and Statistics
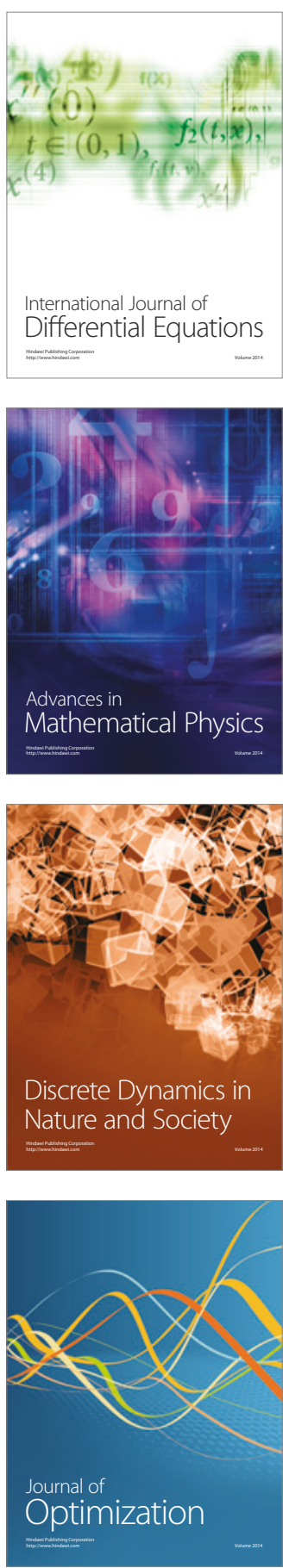